\title{
Low-Noise Parametric Resonant Amplifier
}

\author{
Wooram Lee, Student Member, IEEE, and Ehsan Afshari, Member, IEEE
}

\begin{abstract}
We propose a resonant parametric amplifier with an enhanced noise performance by exploiting the noise-squeezing effect. Noise squeezing occurs through the phase-sensitive amplification process and suppresses one of the two quadrature components of the input noise. When the input signal is only in the direction of the nonsuppressed quadrature component, squeezing can lower that noise figure by almost $3 \mathrm{~dB}$. The resonant structure of the proposed amplifier is inspired by a Fabry-Perot laser amplifier to achieve the squeezing effect using a low number of $L C$ elements. We design and simulate the proposed noise-squeezing parametric amplifier in a conventional $65-\mathrm{nm}$ CMOS process. A minimum noise-squeezing factor of $-0.35 \mathrm{~dB}$ is achieved with a signal gain of $26 \mathrm{~dB}$ for one quadrature component of a 10-GHz narrow-band signal.
\end{abstract}

Index Terms-Distributed system, low-noise amplifier (LNA), noise squeezing, nonlinear capacitor, parametric amplification, phase matching, phase-sensitive gain.

\section{INTRODUCTION}

I N AN RF receiver front end, a low-noise amplifier (LNA) is a critical block since it mainly determines the noise figure (NF) of the entire system. There have been many previous efforts to minimize the NF of LNAs in a CMOS process. A sourcedegenerated CMOS LNA is one of the most prevalent structures, which achieves input matching without a real resistor and exploits an input resonant network for signal amplification [1], [2]. $g_{m}$-boosted and positive feedback LNAs are also attractive modifications of a conventional common-gate CMOS LNA [3], [4]. A sub-0.2-dB NF CMOS LNA was implemented with a non-50- $\Omega$ signal-source impedance [5].

A more exotic approach is to use parametric amplification, in which the gain comes from the nonlinear interaction between the signal and the pump without using transistors. Parametric amplification was actively studied in the 1960s before the transistor technology dominated the integrated circuit design [6]. Recently, several interesting works have revisited parametric amplification for CMOS technology. Discrete-time parametric amplification has been implemented with a MOS varactor to achieve a low-power and low-noise performance for low sampling frequencies [7]-[9]. The parametric process was also exploited for the frequency conversion of a continuous

Manuscript received March 03, 2010; revised May 31, 2010; accepted July 16, 2010. Date of publication November 11, 2010; date of current version February 24, 2011. This work was supported by the C2S2 Focus Center, which is one of the six research centers funded under the Focus Center Research Program, which is a Semiconductor Research Corporation entity. The work of W. Lee was supported by a Samsung fellowship. This paper was recommended by Associate Editor A. Neviani.

The authors are with the School of Electrical and Computer Engineering, Cornell University, Ithaca, NY 14850 USA (e-mail: w1287@cornell.edu).

Digital Object Identifier 10.1109/TCSI.2010.2072370 signal [10]-[12]. However, the parametric amplification cannot provide enough gain for high-frequency signals due to low quality factors of inductors and capacitors in a CMOS process.

In this paper, we propose a parametric amplifier based on a distributed nonlinear resonator to overcome the limitation of low quality factor elements. The distributed nonlinear resonator operates as a regenerative amplifier by supplying the pump amplitude below the oscillation threshold. As a result, the amplifier achieves a high closed-loop gain using a less-than-unity openloop gain [13]. An important property of the proposed amplifier is its phase-sensitive gain, resulting in noise squeezing. One of the quadrature input noise components (e.g., out of phase) is suppressed when the input noise consists of two quadrature components: in phase and out of phase relative to the pump signal. This noise squeezing reduces the amplifier output noise by almost $3 \mathrm{~dB}$ compared to the phase-insensitive amplifier with the same gain. In other words, while a conventional amplifier increases the input noise of both quadratures, the noise-squeezing amplifier increases the noise of one quadrature and, at the same time, decreases the other one.

Noise squeezing was originally studied in optics for precise measurements constrained by the uncertainty principle, which sets a fundamental limit to the simultaneous observation of two conjugate parameters, such as the photon number and its phase [14], [15]. Since the uncertainty principle preserves the multiplication of the variances of two conjugate parameters, the degenerate parametric amplifier can suppress one of the quadrature noise components at the expense of amplifying the other quadrature component through phase-sensitive amplification. Noise squeezing was also demonstrated in the mechanical systems as classical analogues of the optical systems to beat the thermal noise limitation [16], [17]. Finally, Josephson's parametric amplifier using a superconducting quantum interference device was designed to implement noise squeezing in an electrical system [18]-[20]. However, this amplifier requires a very low operation temperature (around $0 \mathrm{~K}$ ) and is not integrable. To the best of our knowledge, our work is the first demonstration of noise squeezing for an LNA in a CMOS process.

The rest of this paper is organized as follows. Section II explains the theory of distributed parametric amplification. Section III discusses the noise-squeezing effect to enhance the noise performance. Section IV explains the pump loss effect, which poses fundamental limits for gain and noise squeezing in a traveling parametric amplifier. Section V proposes a resonant parametric amplifier to overcome the limits mentioned in Section IV and analyzes its phase-sensitive gain and noise-squeezing performance. Section VI summarizes the design procedure and the simulation results. Finally, Section VII concludes our work. 


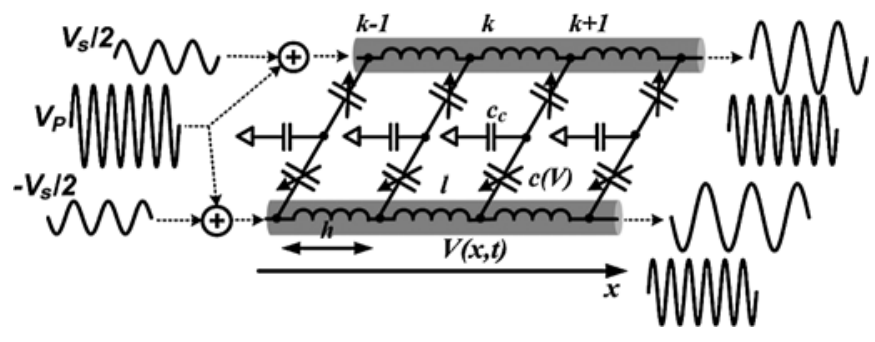

Fig. 1. Parametric amplifier using a differential nonlinear transmission line with dispersion compensation capacitors $C_{c}$. An input signal at frequency $\omega$ is differentially applied, and a pump is injected at a frequency of $2 \omega$ in common mode.

\section{Distributed Parametric Amplification Using A NONLINEAR TRANSMISSION LINE}

In this section, we review the basic theory behind parametric amplification using a nonlinear transmission line.

Assume a uniform artificial differential transmission line that consists of inductors and voltage-dependent (and hence nonlinear) capacitors, as shown in Fig. 1. The nonlinear capacitor is approximated with a first-order function

$$
C(V)=C_{0}(1+b V)
$$

where $C_{0}$ is the capacitance at zero bias and $b$ is the $C / V$ slope. For now, assume that $C_{c}$ is very large, which means that the common node between two lines is ac grounded.

One can easily obtain (2) by applying KCL and KVL and by using approximate partial derivatives with respect to distance $x$ from the beginning of the line, where the voltage on the line is $V(x, t)[21]$. This approximation is valid when the dispersion effect caused by discreetness is negligible due to a small spacing between adjacent $L C$ elements compared to the signal wavelength

$$
\frac{\partial^{2} V}{\partial x^{2}}=L \frac{\partial}{\partial t}\left[C(V) \frac{\partial V}{\partial t}\right]+2 \sqrt{L C_{0}} \alpha \frac{\partial V}{\partial t}
$$

where

$$
\alpha=\frac{1}{2}\left(G Z_{0}+\frac{R}{Z_{0}}\right)
$$

and $L, C, G$, and $R$ are the unit length inductance, capacitance, parasitic conductance, and parasitic resistance, respectively. $Z_{0}$ is the characteristic impedance defined by $\sqrt{L / C_{0}}$.

The pump and the signal are applied to the left end of the transmission line, and the pump frequency is set to twice the signal frequency. Note that the signal is differentially applied between two lines, whereas the pump is in the common mode, as shown in Fig. 1. Then, (2) can be written for a differential signal $V_{s}$

$$
\frac{\partial^{2} V_{s}}{\partial x^{2}}=L C_{0} \frac{\partial^{2} V_{s}}{\partial t^{2}}+L C_{0} b \frac{\partial^{2}\left(V_{s} \cdot V_{p}\right)}{\partial t^{2}}+2 \sqrt{L C_{0}} \alpha_{s} \frac{\partial V_{s}}{\partial t}
$$

where $V_{s}$ and $V_{p}$ are the signal and pump voltages on the transmission line, respectively. $\alpha_{s}$ is the transmission line loss for the signal frequency. We assume that the second-order harmonic generation (SHG) of the pump is sufficiently suppressed since the cutoff frequency of the transmission line is set to lower than twice the pump frequency. The SHG of the signal is canceled out due to the differential signaling in (4).

In (4), the first term on the right corresponds to the linear wave propagation, and the third term represents the loss of the transmission line. The second term on the right represents the nonlinear coupling between the pump and the signal, which results in parametric amplification.

From the coupled-mode theory [22]-[24], the signal can be written as

$$
\begin{aligned}
& V_{s}(x, t)=V_{s}(x) e^{j \omega t}+V_{s}^{*}(x) e^{-j \omega t} \\
& V_{s}(0, t)=A_{s} \cos (\omega t+\phi)
\end{aligned}
$$

where $V_{s}(x)=A(x) e^{-j \beta x}, V_{s}^{*}(x)=A^{*}(x) e^{j \beta x}, \beta$ is the signal propagation constant, and $\phi$ is the initial phase difference between the signal and the pump at $x=0$. Here, "**" denotes the complex conjugate. $A(x)$ and $A^{*}(x)$ are slowly varying functions over $x$, which means $\partial A / \partial x \ll \beta A$.

Assume that the pump is a sinusoidal function at a frequency of $\omega_{p}=2 \omega$. Then, the pump can be written as

$$
\begin{aligned}
V_{p}(x, t) & =A_{p}(x) \cos \left(\omega_{p} t-\beta_{p} x\right) \\
& =\frac{1}{2} A_{p}(x)\left[e^{j\left(\omega_{p} t-\beta_{p} x\right)}+e^{-j\left(\omega_{p} t-\beta_{p} x\right)}\right]
\end{aligned}
$$

where $A_{p}(x)$ is the amplitude of the pump, which is a slowly varying function over $x$, and $\beta_{p}$ is the pump propagation constant.

By substituting (5) and (8) into (4) and by approximating $\beta$ as $\beta \simeq \omega \sqrt{L C_{0}}$ (small dispersion assumption) and $\beta_{p}$ as $\beta_{p}=$ $2 \beta-\Delta \beta$ (the dispersion might not be negligible for the pump, which is at a value that is twice the input frequency), we derive the active coupled-mode equations for $A(x)$ and $A^{*}(x)$ as

$$
\begin{aligned}
\frac{\partial A}{\partial x} & =-\frac{j \beta b A_{p}}{4} A^{*} e^{j \Delta \beta x}-\alpha_{s} A \\
\frac{\partial A^{*}}{\partial x} & =\frac{j \beta b A_{p}}{4} A e^{-j \Delta \beta x}-\alpha_{s} A^{*} .
\end{aligned}
$$

For now, $A_{p}$ is assumed to be constant over $x$ to obtain an analytic solution of (9) and (10). This assumption means that we neglect the pump loss in the transmission line and the transferred pump energy to the signal frequency. Under this assumption, the general solution for (9) and (10) is

$$
A=e^{\left(-\alpha_{s}+j \Delta \beta / 2\right) x}\left(A_{1} e^{s x}+A_{2} e^{-s x}\right)
$$

where

$$
s=\sqrt{\left(\frac{\beta b A_{p}}{4}\right)^{2}-\left(\frac{\Delta \beta}{2}\right)^{2}}
$$

and $A_{1}$ and $A_{2}$ are constants over $x$.

By applying the boundary condition of (6)-(9), the complete solution is obtained as

$$
V_{s}(x, t)=A_{s} e^{-\alpha_{s} x}\left[C_{1} \cos (\omega t-\beta x)+S_{1} \sin (\omega t-\beta x)\right]
$$

where

$$
\begin{aligned}
& C_{1}(x, \phi)=\cosh \left(s_{0} x\right) \cos \phi-\sinh \left(s_{0} x\right) \sin \phi \\
& S_{1}(x, \phi)=\sinh \left(s_{0} x\right) \cos \phi-\cosh \left(s_{0} x\right) \sin \phi
\end{aligned}
$$


and $s_{0}=\beta b A_{p} / 4$ is the first term on the right side of (12). It is noteworthy that, in (13), we have assumed no dispersion for the pump $(\Delta \beta=0)$. At the end of this section, we will introduce a method to compensate the dispersion for the pump, validating this assumption.

From (13), the parametric gain is

$$
G(x)=\frac{\left|V_{s}(x)\right|}{A_{s}}=e^{-\alpha_{s} x} \sqrt{\cosh \left(2 s_{0} x\right)-\sinh \left(2 s_{0} x\right) \sin 2 \phi} .
$$

For the maximum and minimum gains, the signal is exponentially growing or decaying over $x$

$$
G(x)= \begin{cases}e^{\left(s_{0}-\alpha_{s}\right) x}, & \text { for } \phi=-\frac{\pi}{4}(\max ) \\ e^{-\left(s_{0}+\alpha_{s}\right) x}, & \text { for } \phi=\frac{\pi}{4}(\min )\end{cases}
$$

Equation (16) can be converted into the gain equation for the section number $k$ of the discrete transmission line by inserting $L=l / h, C_{0}=c_{0} / h, G=g / h, R=r / h$, and $x=k h$, where $h$ is the spacing between two adjacent $L C$ sections. $l, c_{0}, g$, and $r$ are the inductance, average varactor capacitance, conductance, and resistance for one $L C$ section, respectively. Using

$$
\begin{aligned}
s_{0} x & =\frac{b A_{p}}{4} \cdot \omega \sqrt{\left(\frac{l}{h}\right)\left(\frac{c_{0}}{h}\right)} \cdot k h=\frac{b A_{p} \omega \sqrt{l c_{0}}}{4} \cdot k \\
\alpha_{s} x & =\frac{1}{2}\left(g Z_{0}+\frac{r}{Z_{0}}\right) \cdot k
\end{aligned}
$$

the parametric gain for the $k$-section nonlinear transmission line is presented by

$$
G_{k}=G(k h)=e^{-\widetilde{\alpha_{s}} k} \sqrt{\cosh \left(2 \widetilde{s_{0}} k\right)-\sinh \left(2 \widetilde{s_{0}} k\right) \sin 2 \phi}
$$

where

$$
\begin{aligned}
\widetilde{s_{0}} & =\frac{b A_{p} \omega \sqrt{l c_{0}}}{4} \\
\widetilde{\alpha_{s}} & =\frac{1}{2}\left(g Z_{0}+\frac{r}{Z_{0}}\right) .
\end{aligned}
$$

Fig. 2 shows the plot of the calculated parametric gain for $20 L C$ sections based on (20) to show the effect of the initial phase differences between the pump and the signal for different nonlinear factors, defined by $b A_{p}$. The input and pump frequencies are 10 and $20 \mathrm{GHz}$, respectively. The cutoff frequency, defined by $\omega_{c}=2 / \sqrt{l c_{0}}$, is set to $25 \mathrm{GHz}$, and the characteristic impedance is $50 \Omega$. We assume that the quality factor of the transmission line for a signal frequency is 10 , which corresponds to $\widetilde{\alpha_{s}}=0.04 / \mathrm{section}[25]$. As the nonlinear factor increases, the gain plot shows a higher amplification and attenuation depending on the phase difference. The phase difference between the maximum and minimum is $\pi / 2$, which clearly shows the phase-sensitive gain for quadrature signals.

Intuitively, at each section of the line, the nonlinear interaction between the signal at $\omega$ and the pump at $2 \omega$ generates two frequency components at $3 \omega$ and $\omega$. The $3 \omega$ component is suppressed by the cutoff frequency of the transmission line, and the $\omega$ component is added to the signal. However, this adding

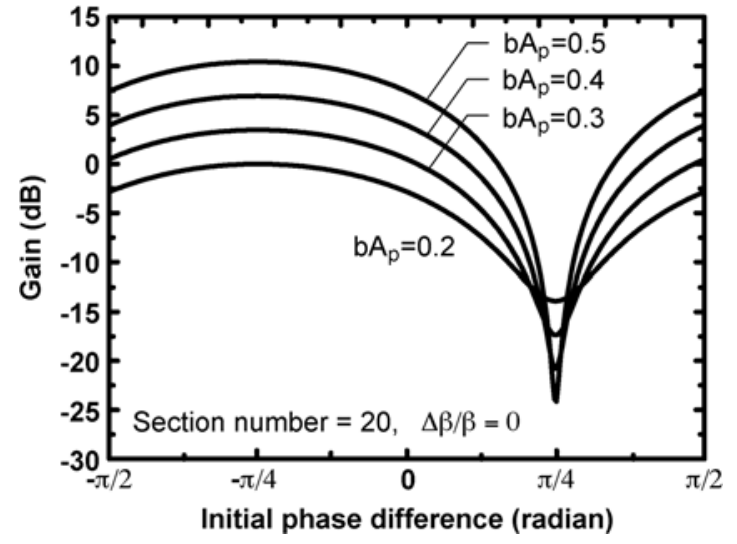

Fig. 2. Calculated gain versus the initial phase difference between the pump and the signal for different nonlinearity factors $\left(l=630 \mathrm{pH}, c_{0}=250 \mathrm{fF}\right.$, and $\omega=10 \mathrm{GHz}$ ).

process can be constructive or destructive, depending on the phase difference between the signal and the pump. A series of this process over multiple sections enables the gain to exponentially grow (energy transfer from the pump to the signal) or to be attenuated (energy transfer from the signal to the pump). When the pump frequency is exactly twice the signal frequency, parametric amplification is called as degenerate, mainly characterized by its phase-sensitive gain.

Note that the exponent $s_{0}$ in (17) is proportional to the propagation constant $\beta$ as well as to the varactor $C / V$ slope and the pump amplitude. This is because the increase in the propagation constant is equivalent to the increase in the effective transmission line length. However, the increase in the propagation constant also lowers the cutoff frequency and decreases the pump amplitude. Therefore, the propagation constant should be carefully selected.

The aforementioned analysis neglects the effect of dispersion $(\Delta \beta=0)$. However, the discreetness of the artificial transmission line causes nonnegligible dispersion and results in an undesired phase shift between the pump and the signal, weakening the parametric coupling between them, as shown in Fig. 3. Fig. 4 shows how the propagation constant mismatch degrades the parametric gain. In addition to the drop in the exponential constant in (12), an even more serious effect comes from the accumulation of the phase mismatch due to the differences in the propagation constant. A small phase mismatch is accumulated over multiple sections to reach a certain amount of phase difference between the pump and the signal, causing attenuation instead of amplification and limiting a maximum gain to the curve of the gain plot over the section number.

To maintain $\Delta \beta=0$, we have introduced the dispersion compensation capacitor $C_{c}$ at the common node, as shown in Fig. 1. This capacitor only affects the common-mode pump because the differential signal does not see it due to the virtual ground in the middle node. With the dispersion compensation capacitor, the net average capacitance for the pump drops to $C_{0}$ in series with $C_{c} / 2$, decreasing the pump propagation delay without changing the signal propagation. By selecting the right value for $C_{c}$, one can completely phase match the signal and pump. 


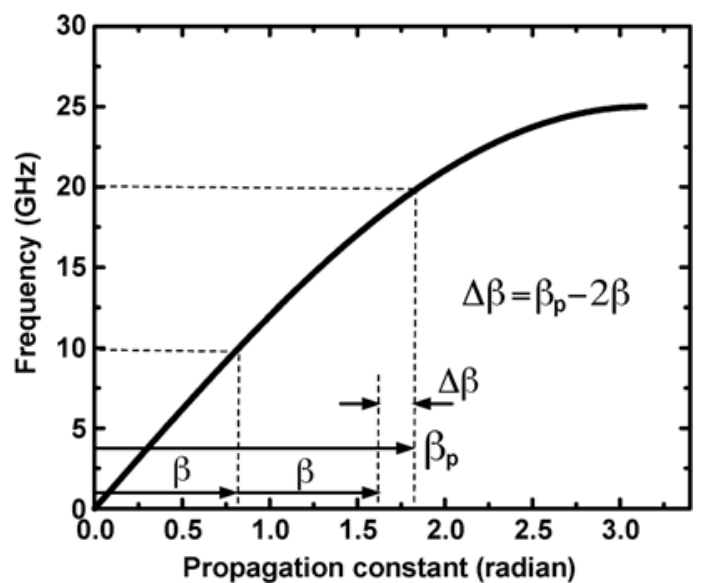

Fig. 3. Dispersion relation of an artificial transmission line when the pump frequency $(20 \mathrm{GHz})$ is comparable to the cutoff frequency $(25 \mathrm{GHz})$.

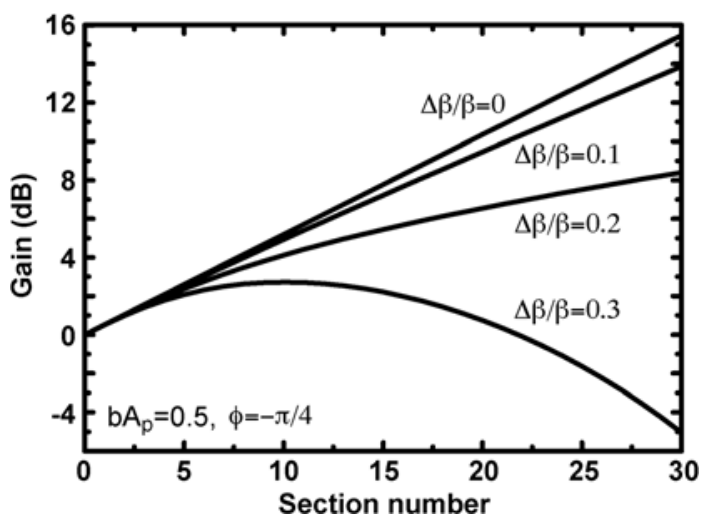

Fig. 4. Calculated gain versus the section number for different propagation constant mismatches between the pump and the signal $\left(l=630 \mathrm{pH}, c_{0}=\right.$ $250 \mathrm{fF}$, and $\omega=10 \mathrm{GHz}$ ).

\section{Noise OF THE DEgENERATE PARAMETRIC AMPLIFIER}

Since the degenerate parametric process uses only reactive components, potentially, it can achieve a better NF than the conventional transistor-based amplifiers. In addition, its phasesensitive gain shows an interesting property: noise squeezing. The equipartition theorem suggests that the input noise (usually, thermal noise) is circularly symmetric for two quadrature components in a thermal equilibrium, and it can be written as a narrow-band representation of the carrier (signal) frequency $\omega[26],[27]$

$$
\begin{aligned}
n(t) & =n_{I}(t) \cos \omega t+n_{Q}(t) \sin \omega t \\
\overline{n_{I}^{2}(t)} & =\overline{n_{Q}^{2}(t)} \cong \sigma^{2}
\end{aligned}
$$

where $n_{I}(t)$ and $n_{Q}(t)$ are the slowly varying functions compared to a sinusoidal function at $\omega$.

When $n(t)$ is injected into a degenerate parametric amplifier with $k$-section lossless nonlinear transmission line $\left(\widetilde{\alpha_{s}}=0\right)$ pumped at $2 \omega$ and when the pump has the right phase so that $n_{I}(t)$ experiences the maximum gain, then $n_{Q}(t)$ has the maximum attenuation based on (17)

$$
\overline{n_{I}^{2}(t)_{\mathrm{out}}}=\left|G_{k, \phi=-\pi / 4}\right|^{2} \overline{n_{I}^{2}(t)}=F_{k} \sigma^{2}
$$

$$
\overline{n_{Q}^{2}(t)_{\text {out }}}=\left|G_{k, \phi=\pi / 4}\right|^{2} \overline{n_{Q}^{2}(t)}=\frac{1}{F_{k}} \sigma^{2}
$$

where the in-phase power gain for $k$-section lossless nonlinear transmission line (NLTL) $\left(F_{k}\right)$ is defined as

$$
F_{k}=e^{2 \widetilde{s_{0} k}} .
$$

The multiplication of the standard deviations of $n_{I}(t)_{\text {out }}$ and $n_{Q}(t)_{\text {out }}$ is given by

$$
\begin{aligned}
\left.{\sqrt{\overline{n_{I}^{2}(t)}}}_{\text {out }} \cdot \sqrt{\overline{n_{Q}^{2}(t)}}\right)_{\text {out }} & =\sqrt{F_{k}} \sigma \cdot \frac{1}{\sqrt{F_{k}}} \sigma=\sigma^{2} \\
& =\sqrt{\overline{n_{I}^{2}(t)}} \cdot \sqrt{\overline{\overline{n_{Q}^{2}(t)}}} .
\end{aligned}
$$

Equation (29) shows that the multiplication of two quadrature noise components is preserved since one quadrature noise component (out of phase) is suppressed at the expense of amplifying the other quadrature noise component (in phase) through phase-sensitive amplification.

Here, we introduce a noise-squeezing factor $S_{F}$, which is equal to NF when the information is placed only in the single quadrature phase. This needs to be differentiated with the general NF, which usually considers information in two quadrature phases. Assuming that the thermal noise caused by power dissipation on the transmission line is negligible and that the signal information is only in the in-phase direction, the noisesqueezing factor of the parametric amplifier is defined as

$$
S_{F}=\frac{S N R_{\mathrm{IN}}}{S N R_{\mathrm{OUT}}}=\frac{\frac{v_{s}^{2}}{n_{I}^{2}+n_{Q}^{2}}}{\frac{F_{k} v_{s}^{2}}{F_{k} n_{I}^{2}+1 / F_{k} n_{Q}^{2}}}=\frac{1}{2}\left(1+\frac{1}{F_{k}^{2}}\right)
$$

where $n_{I}^{2}=n_{Q}^{2} \cong \sigma^{2}$ and $v_{s}$ is a signal amplitude.

Equation (30) shows that the squeezing factor approaches -3 $\mathrm{dB}$ as $F_{k}$ increases. In other words, the noise is redistributed from a circular to an oval shape through parametric amplification to have a higher SNR in one quadrature direction. Fig. 5 shows the time-domain effect of the noise squeezing and the noise distribution over the phase. The output of the parametric amplifier can be written as

$$
\begin{aligned}
v_{\text {out }}(t) & =\sqrt{F_{k}} v_{s}(t) \cos (\omega t-\theta)+n_{\text {out }}(t) \\
& =\sqrt{F_{k}}\left[v_{s}(t)+n_{I}(t)\right] \cos (\omega t-\theta-\zeta)
\end{aligned}
$$

where

$$
\zeta=\tan ^{-1}\left(\frac{n_{Q}(t)}{F_{k} v_{s}}\right) \cong \frac{n_{Q}(t)}{F_{k} v_{s}}
$$

and $\theta$ is the phase shift due to the propagation delay over the transmission line. Equation (32) shows that $n_{I}(t)$ contributes to the amplitude fluctuation, whereas $n_{Q}(t)$ contributes to the phase fluctuation. Equations (32) and (33) explain how the squeezing suppresses the phase (timing) fluctuation at the output by increasing the amplitude fluctuation with the same ratio, as shown in Fig. 5(c). This is fundamentally different from a linear amplifier (phase-insensitive amplifier) that provides the same amount of amplification for two quadrature 

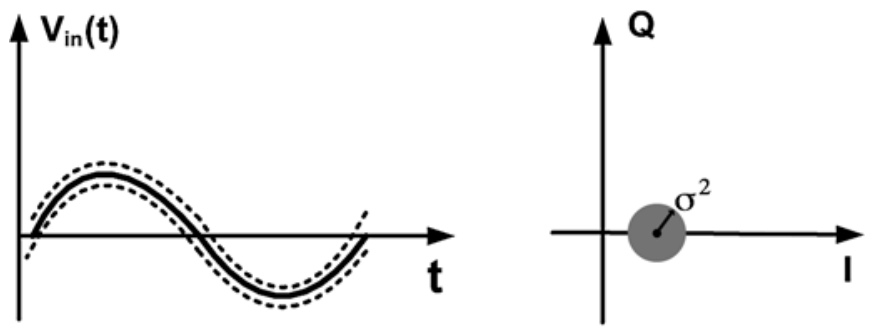

(a)
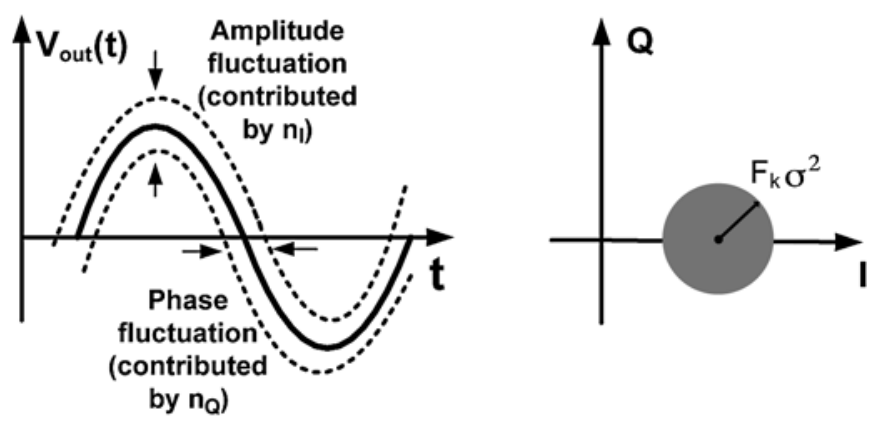

(b)
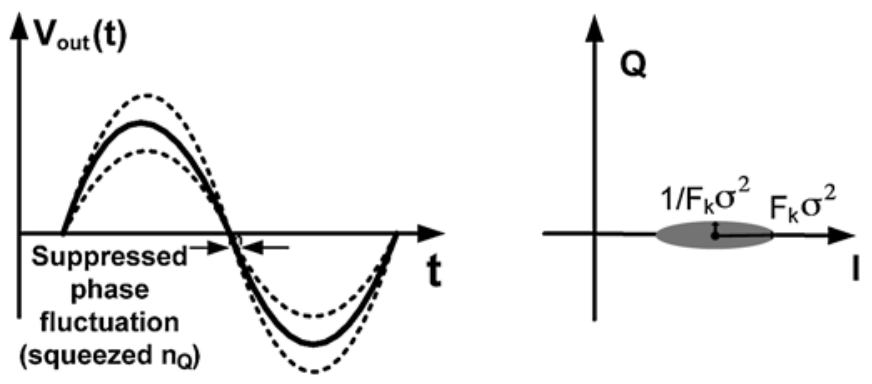

(c)

Fig. 5. Observation of the squeezed output noise by phase-sensitive amplification in a time and phase domain compared to linear amplification. (a) Input signal. (b) Output signal through linear amplification. (c) Output signal through phase-sensitive amplification. To simplify the notations, the phase of the pump is shifted by $\pi / 2$. In (17), the signal has its maximum and minimum at $\phi=0$ and $\phi=\pi / 2$, respectively.

components, resulting in a circularly symmetric output noise distribution, as shown in Fig. 5(b).

Next, we take into account the effect of the transmission line loss in the parametric amplifier squeezing factor $S_{F}$. The parametric amplifier consists of two transmission lines that generate two independent noises $\left(v_{n 1 i}\right.$ and $\left.v_{n 2 i}\right)$ at the $i$ th node. Because only the differential signal is amplified, the output noise contributed by the power dissipation at the $i$ th node is

$$
\begin{aligned}
\left.\overline{v_{n \text { out }}^{2}}\right|_{i} & =\frac{e^{-2 \widetilde{\alpha_{s}}(k-i)}}{2}\left(F_{k-i}+\frac{1}{F_{k-i}}\right)\left(\overline{v_{n 1 i}^{2}}+\overline{v_{n 2 i}^{2}}\right) \\
& =2 e^{-2 \widetilde{\alpha_{s}}(k-i)} \cosh \left[2 \widetilde{s_{0}}(k-i)\right] \overline{v_{n 1 i}^{2}} .
\end{aligned}
$$

The thermal noise power generated at the $i$ th node that travels toward the output is

$$
\begin{aligned}
\overline{v_{n 1 i}^{2}} & =k T B r+k T B g Z_{0}^{2} \\
& =2 k T B Z_{0}\left(\frac{r}{2 Z_{0}}+\frac{g Z_{0}}{2}\right)=2 k T B Z_{0} \widetilde{\alpha_{s}}
\end{aligned}
$$

where $B$ is the bandwidth over which the noise is measured. $r$ and $g$ are the parasitic series resistance and parallel conductance which represent the inductor and varactor losses, respectively. Combining (35) and (37), the total output noise associated with the transmission line loss is given by

$$
\overline{v_{\text {nout }}^{2}}=4 k T B Z_{0} \widetilde{\alpha_{s}} \sum_{i=1}^{k} e^{-2 \widetilde{\alpha_{s}} i} \cosh \left(2 \widetilde{s_{0}} i\right) .
$$

From (30) and (38), the noise-squeezing factor becomes

$$
S_{F}=\frac{1}{2}\left(1+\frac{1}{F_{k}^{2}}\right)+\frac{2 \widetilde{\alpha_{s}}}{F_{k}} \sum_{i=1}^{k} e^{2 \widetilde{\alpha_{s}}(k-i)} \cosh \left(2 \widetilde{s_{0}} i\right)
$$

where the input signal source impedance is matched with the characteristic impedance $Z_{0}$. In the absence of the pump $\left(F_{k}=\right.$ 1), from (39), the NF or the squeezing factor is simply approximated with $e^{2 \widetilde{\alpha_{s}} k}$, which is the reciprocal of the transmission line loss and is expected from a conventional transmission line.

Fig. 6 shows the calculated squeezing factor based on (39). The signal and pump frequencies are 10 and $20 \mathrm{GHz}$, respectively. The cutoff frequency is set to $25 \mathrm{GHz}$. When the transmission line is lossless, the amplifier squeezing factor approaches $-3 \mathrm{~dB}$ for large nonlinear factors, as shown in Fig. 6(a). However, the transmission line loss significantly degrades the squeezing factor. The phase sensitivity of the squeezing factor is also investigated, as shown in Fig. 6(b). Since the input noise distribution is assumed to be circularly symmetric and independent of the input signal, the variation of the squeezing factor is caused only by a change in the signal gain. The squeezing factor is also calculated as a function of the section number, as shown in Fig. 6(c). As the section number increases, the squeezing effect exponentially increases, resulting in a better squeezing factor. However, in the presence of transmission loss, the number of noise sources also increases with the section number. Therefore, for a given nonlinearity, an optimum number of sections will result in a minimum squeezing factor.

\section{EFFECT OF PUMP Loss}

The previous sections examined the signal gain and noise performance in the absence of pump attenuation for an analytical solution. However, pump loss is critical since the signal gain is an exponential function of the pump amplitude, as shown in (17).

Fig. 7 shows the simulated pump loss effect on the gain and squeezing factor based on (9) and (10) when the phase difference between the signal and the pump is $-\pi / 4$ for a maximum gain. When the pump loss is negligible, the log-scale gain is linearly proportional to the section number, following (17). However, as the pump loss increases, the amplifier gain reaches a peak for a certain number of sections. Before this point, since the pump amplitude is large, the gain increases as the signal propagates. After this point, the pump amplitude is too low to compensate the loss of the transmission line, resulting in a lower gain. For a higher pump loss, this optimal number of sections also decreases, thereby resulting in a lower gain peak. Considering that the typical value of the quality factor of the transmission line is around 10 for a pump frequency of $20 \mathrm{GHz}$, the maximum gain is only $5 \mathrm{~dB}$, with 22 sections. One might consider 


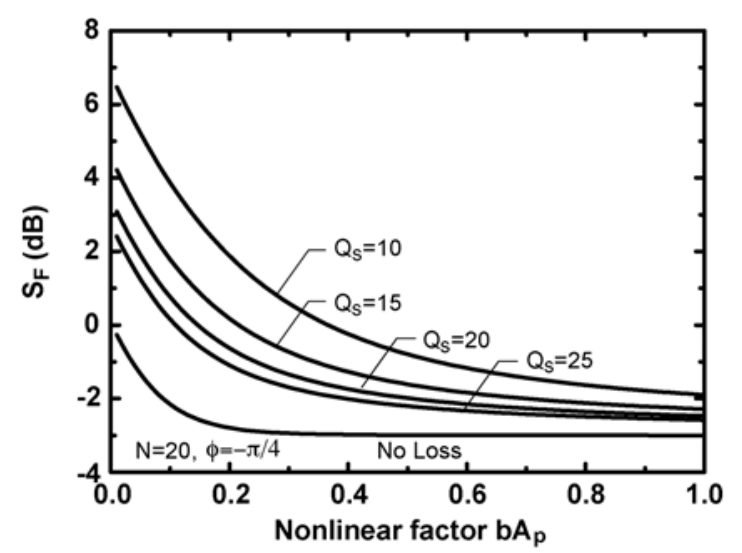

(a)

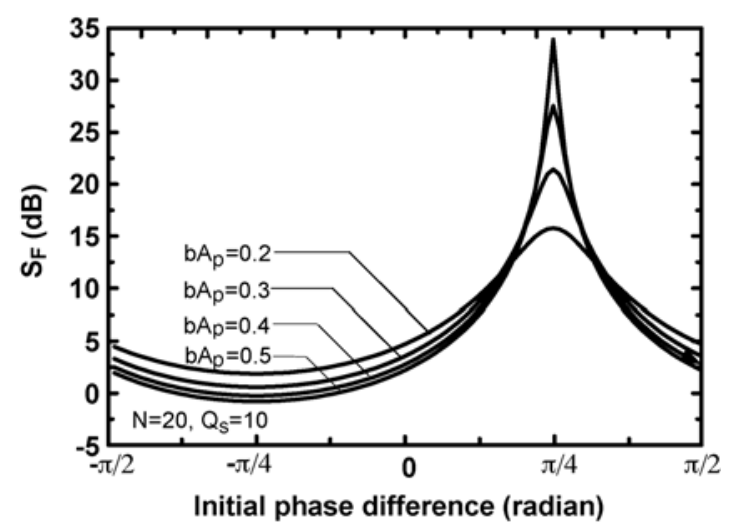

(b)

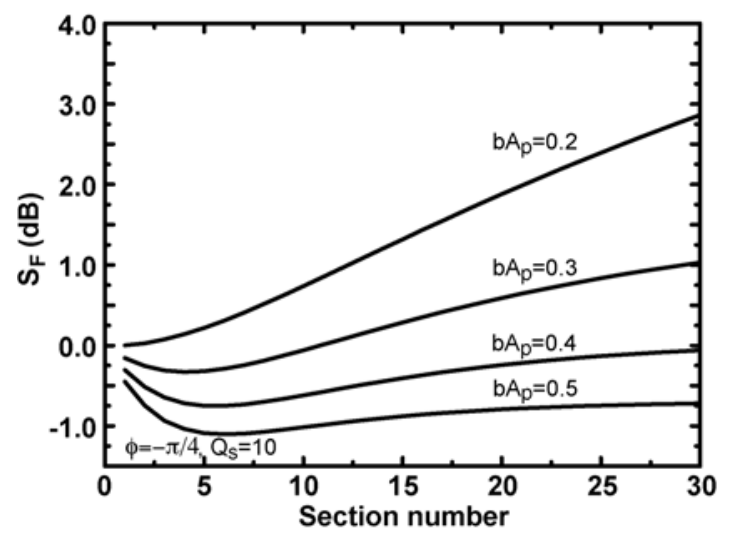

(c)

Fig. 6. Calculated squeezing factor versus the (a) nonlinear factor for different transmission line loss, (b) initial phase difference between the pump and the signal for different nonlinear factors, and (c) section number for different nonlinear factors $\left(l=630 \mathrm{pH}, c_{0}=250 \mathrm{fF}\right.$, and $\left.\omega=10 \mathrm{GHz}\right)$.

injecting the pump frequency at multiple points of the transmission line to compensate the loss. Unfortunately, this method would require a higher pump power and would also result in a more complex design and a higher footprint.

Pump loss also degrades the squeezing factor: loss results in pump attenuation which, in turn, translates to a lower squeezing effect. This is shown in Fig. 7(b), where the squeezing factor increases with the section number.

\section{RESONANT PARAMETRIC AMPLIFIER}

To overcome the challenges of the traveling-wave structures associated with a large number of lumped $L C$ elements and

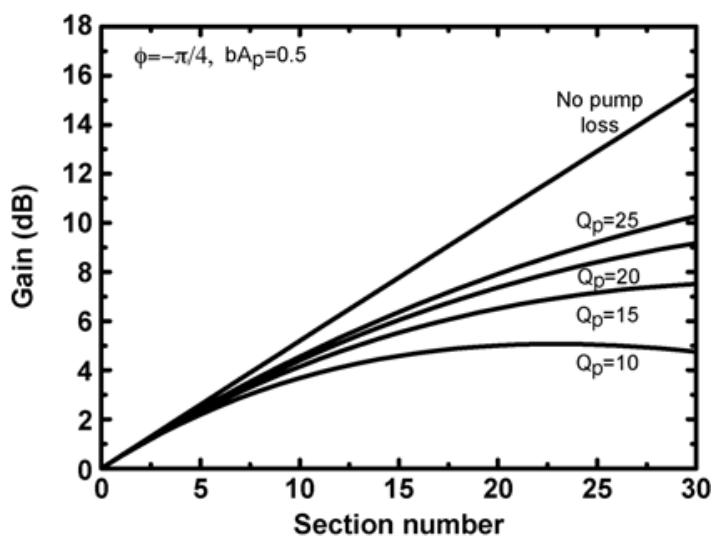

(a)

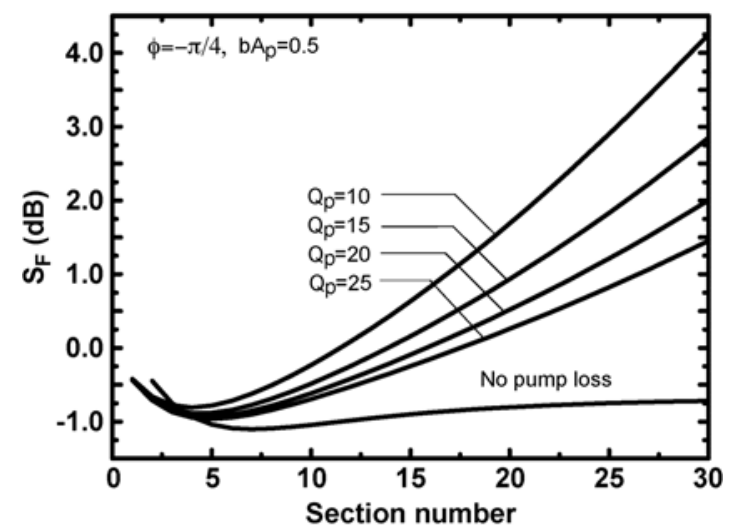

(b)

Fig. 7. Simulated pump loss effect on the (a) gain and (b) squeezing factor versus the section number for different pump losses $\left(l=630 \mathrm{pH}, c_{0}=250 \mathrm{fF}\right.$, $Q_{s}=10$, and $\omega=10 \mathrm{GHz}$ ).

pump loss due to a limited quality factor, we propose a resonant regenerative amplifier based on a parametric oscillator structure.

\section{A. Review of the Reflective Parametric Oscillator}

Fig. 8 shows the reflective parametric oscillator and its standing wave formation for the signal and pump frequencies [12]. Parametric oscillation occurs when the parametric amplification compensates the loss of a resonator at the resonance frequency. The resonator consists of two transmission lines with four phase-matched $L C$ sections that are connected at both ends. Oscillation starts from the ambient thermal noise of the resonator when the pump is strong enough to compensate for the loss. The noise component grows by traveling back and forth between two reflective ends through the degenerate parametric amplification, finally generating a stable oscillation signal at a half-pump frequency.

The upper part of Fig. 8 shows different standing wave formations inside the resonator for both the pump and the signal. The differential signal sees the resonator ends as shorts due to the virtual ground, while the common-mode pump sees the ends as open nodes. The effective length of the four- $L C$-section resonator is equal to a pump wavelength $\lambda_{p}$, which is half of the signal wavelength $\lambda_{s}$. With the reflection at both ends, the signal forms a $\lambda_{s} / 2$ standing wave with minimum amplitudes at the two boundaries. On the other hand, the pump forms a $\lambda_{p}$ standing wave with maximum amplitudes at both ends. The higher modes for the pump and the signal are suppressed by the 


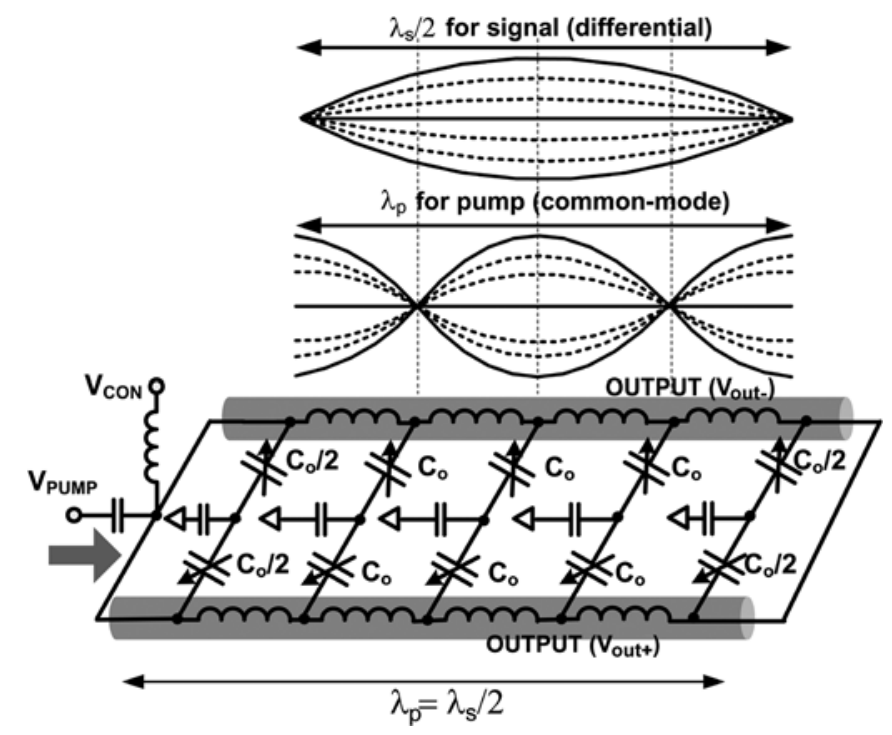

Fig. 8. Reflective distributed parametric oscillator and its standing wave formation for the signal and pump frequencies.

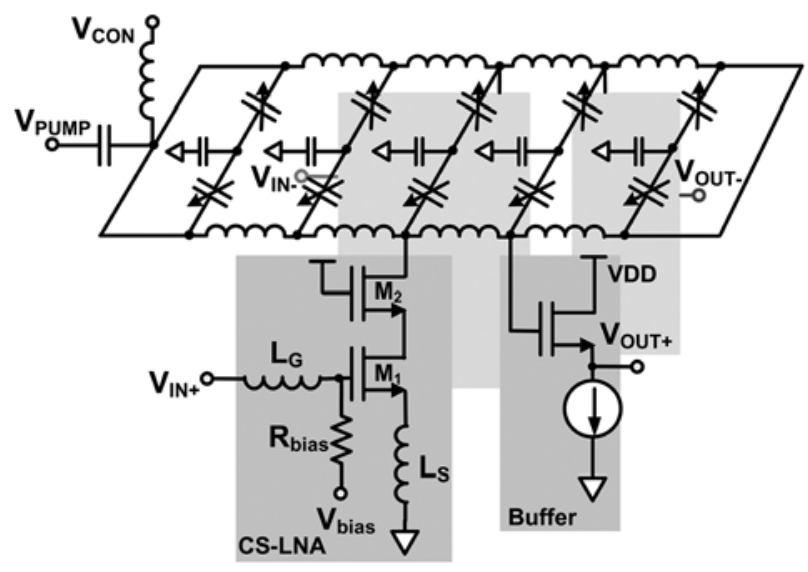

Fig. 9. Differential parametric resonant amplifier with an output buffer.

resonator cutoff frequency. The output port is selected at the " $3 / 4 \lambda_{p}$ " location to suppress the pump signal by exploiting the different standing wave formations for the signal and the pump.

\section{B. Regenerative Amplifier With a Parametric Resonator}

By operating the parametric oscillator with a pump below the oscillation threshold, another form of degenerate parametric amplification can be achieved, as shown in Fig. 9. Based on the resonator structure in Fig. 8, the input signal is applied to the middle of the resonator through the drain of a common-source LNA. The source followers are simply used as output buffers to drive a $50-\Omega$ output load. The control voltage $V_{\text {con }}$ enables us to tune the resonant frequency.

We can intuitively explain the principle of the resonant parametric amplifier by comparing it with a conventional regenerative resonant amplifier that has been recently proposed for the high quality factor bandpass filtering [28]. As shown in Fig. 10(a), a negative resistance in a resonator compensates the loss and results in a higher quality factor. This increased quality

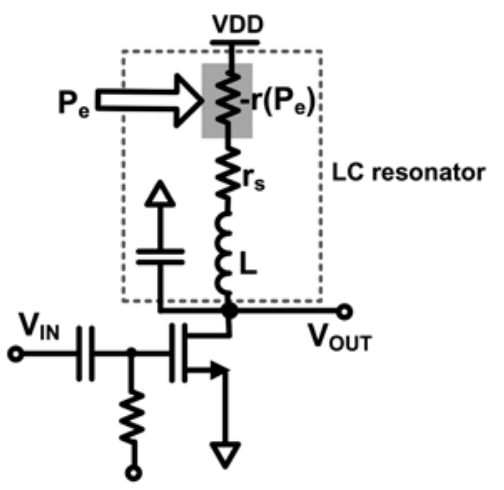

(a)

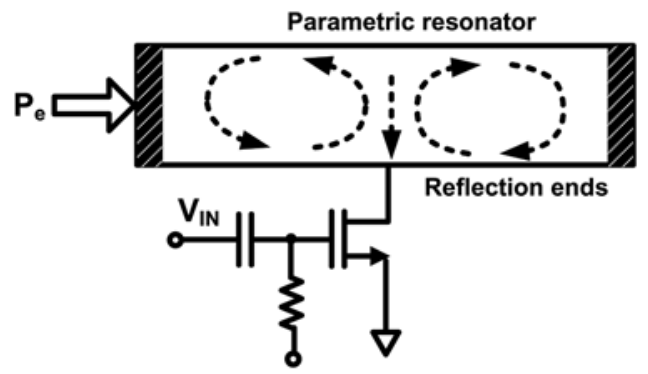

(b)

Fig. 10. (a) Conventional resonant amplifier with a negative resistor. (b) Amplification using a parametric resonator with a pump level below the oscillation threshold.

factor provides a larger output impedance around the resonance frequency given by $Z_{\text {out }}=Q^{2} r_{\text {eff }}$, resulting in a higher gain

$$
G=g_{m} Z_{\mathrm{out}}=\frac{g_{m} L}{C r_{s}} \frac{1}{1-r\left(P_{e}\right) / r_{s}}
$$

where $g_{m}$ is the transconductance of the transistor, $r_{s}$ is a series resistance modeling the loss of the resonator, $L$ is the inductance of the resonator, and $-r\left(P_{e}\right)$ is the negative resistance generated by the external power $P_{e}$. We can clearly observe the similarity between (40) and the parametric gain, which will be presented in (51). Note that, if the negative resistance $-r\left(P_{e}\right)$ is larger than the resonator loss $r_{s}$, the amplifier turns into an $L C$ oscillator. The same happens to the parametric amplifier when the pump power is more than the oscillation threshold.

However, the proposed amplifier does not use the extra transistor to compensate for the resonator loss since the gain comes from the parametric amplification. More importantly, the proposed amplifier provides the phase-sensitive gain for the noise squeezing at the cost of its complicated structure.

For theoretical analysis, we consider the coupled-mode equations of (9) and (10). Adding terms that represent the injection of the input signal and pump into the resonator, these coupled-mode equations are modified to

$$
\begin{aligned}
\frac{\partial A}{\partial x} & =-\frac{j \beta b A_{p}}{4} A^{*}-\alpha_{s} A+\kappa_{s} \frac{A_{s}}{2} e^{j \phi} \\
\frac{\partial A^{*}}{\partial x} & =\frac{j \beta b A_{p}}{4} A-\alpha_{s} A^{*}+\kappa_{s} \frac{A_{s}}{2} e^{-j \phi} \\
\frac{\partial A_{p}}{\partial x} & =-\alpha_{p} A_{p}+\kappa_{p} A_{\mathrm{pin}}
\end{aligned}
$$


where $\kappa_{s}$ and $\kappa_{p}$ represent the increase ratios in the signal and pump amplitudes inside the resonator due to the injected signal and pump, respectively. The significance of this term will be seen when the reflections are taken into account inside the resonator, which will be presented in (47) and (48). $A_{s}$ and $A_{\text {pin }}$ are the signal and pump amplitudes that enter the resonator, respectively.

By the structural symmetry of the resonator, we can only consider the left half of the resonator for the following analysis. It is noteworthy that, even though the pump is only applied to the left end of the resonator, for our analysis, we can imagine a virtual pump injection from the right end. This is due to the fact that the resonator length is $\lambda_{p}$, which means that the two ends are in phase for the pump frequency.

For the steady-state response, $\partial A / \partial x, \partial A^{*} / \partial x, \partial A_{p} / \partial x=$ 0 . Combining (41) and (42) to eliminate $A^{*}$, the signal amplitude on the resonator $|A|$ becomes

$$
|A|=\frac{\kappa_{s} A_{s}}{2\left(\alpha_{s}^{2}-s_{0}^{2}\right)} \sqrt{s_{0}^{2}+\alpha_{s}^{2}-2 s_{0} \alpha_{s} \sin 2 \phi}
$$

where

$$
s_{0}=\frac{\beta b A_{p}}{4}=\frac{\beta b \kappa_{p}}{4 \alpha_{p}} A_{\text {pin }} .
$$

At the resonator end where the pump is injected, the relation between the $m$ th and $(m+1)$ th round-tripped pump amplitudes $\left(A_{p_{m}}\right.$ and $\left.A_{p_{m+1}}\right)$ is

$$
A_{p_{m+1}}=A_{p_{m}} \exp \left(-2 \alpha_{p} d\right)+A_{\text {pin }}
$$

where $2 d$ is the round-trip length of the resonator. Inserting $A_{p_{m+1}}=A_{p_{m}}=\left.A_{p}\right|_{\text {steady }}$ and $\left.A_{p}\right|_{\text {steady }}=\kappa_{p} A_{\text {pin }} / \alpha_{p}$ into (46), $\kappa_{p}$ is reduced to

$$
\kappa_{p}=\frac{\alpha_{p}}{\left(1-\exp \left(-2 \alpha_{p} d\right)\right)} .
$$

In a similar way, $\kappa_{s}$ can be obtained as

$$
\kappa_{s}=\frac{\alpha_{s}}{1-\exp \left(-\alpha_{s} d\right)}
$$

By rearranging (44)

$$
G_{\phi}=\frac{|A|}{A_{s}}=\frac{\kappa_{s} \sqrt{\left(\frac{A_{\mathrm{pin}}}{A_{\mathrm{th}}}\right)^{2}-2\left(\frac{A_{\mathrm{pin}}}{A_{\mathrm{th}}}\right) \sin 2 \phi+1}}{2 \alpha_{s}\left[1-\left(A_{\mathrm{pin}} / A_{\mathrm{th}}\right)^{2}\right]}
$$

where

$$
A_{\mathrm{th}}=\frac{4 \alpha_{s} \alpha_{p}}{\beta b \kappa_{p}}=\frac{2\left(1-\exp \left(-2 \alpha_{p} d\right)\right)}{Q_{p} b} .
$$

From (49), when the pump amplitude is below the oscillation threshold $\left(A_{\mathrm{pin}}<A_{\mathrm{th}}\right)$, the maximum and minimum gains are

$$
G_{\phi}= \begin{cases}\frac{\kappa_{s}}{2 \alpha_{s}}\left(1-\left(\frac{A_{\mathrm{pin}}}{A_{\mathrm{th}}}\right)\right)^{-1}, & \text { for } \phi=-\frac{\pi}{4}(\max ) \\ \frac{\kappa_{s}}{2 \alpha_{s}}\left(1+\left(\frac{A_{\mathrm{pin}}}{A_{\mathrm{th}}}\right)\right)^{-1}, & \text { for } \phi=\frac{\pi}{4}(\min ) .\end{cases}
$$

Based on (51), when the pump power is just below the threshold, the amplifier has the lowest gain (i.e., maximum attenuation for $\phi=\pi / 4)$, which is half the gain in the absence of the pump. This sets a fundamental limit of a maximum squeezing ratio of $6 \mathrm{~dB}$ in the proposed system. Next, we are going to calculate the total gain of the parametric amplifier. The voltage gain of a source-degenerated amplifier that injects the signal into the resonator, shown in Fig. 9, is [1]

$$
G_{0}=\frac{A_{s}}{V_{\mathrm{IN}}}=\frac{\omega_{T} Z_{0}}{2 \omega R_{s}}
$$

where $V_{\mathrm{IN}}$ is the input amplitude, $\omega_{T}$ is the cutoff frequency of $M_{1}, Z_{0}$ is the characteristic impedance of the resonator, $R_{s}$ is the signal source impedance, and $\omega$ is the signal frequency, assuming that the input matching is achieved using a sourcedegenerated inductor $L_{s}$.

Combining (49) and (52), the total gain of the parametric resonant amplifier becomes

$$
\begin{aligned}
G_{T} & =\frac{A_{s}}{V_{\mathrm{IN}}} \cdot \frac{|A|}{A_{s}} \cdot \gamma=G_{0} G_{\phi} \gamma \\
& =G_{T 0} \frac{\sqrt{\left(\frac{A_{\mathrm{pin}}}{A_{\mathrm{th}}}\right)^{2}-2\left(\frac{A_{\mathrm{pin}}}{A_{\mathrm{th}}}\right) \sin 2 \phi+1}}{\left[1-\left(\frac{A_{\mathrm{pin}}}{A_{\mathrm{th}}}\right)^{2}\right]}
\end{aligned}
$$

where $\gamma$ is the voltage increase ratio due to the standing wave formation, defined by $\gamma=2|\sin (\beta x)|(\gamma \cong 1.41$ for the output taken out at $3 / 4 \lambda_{p}$ ) [25]. $G_{T 0}$ is the gain without the pump injection, defined by $G_{T 0}=\kappa_{s} \gamma G_{0} / 2 \alpha_{s}$.

Fig. 11(a) shows the calculated gain enhancement ratio $G_{T} / G_{T 0}$ due to the parametric amplification with respect to the phase difference between the signal and the pump for different pump amplitudes based on (54). As the pump amplitude approaches the oscillation threshold, the gain plot shows higher amplification and attenuation depending on the phase difference. The phase difference between the maximum and minimum is $\pi / 2$, which clearly shows the phase-sensitive gain for quadrature signals. Fig. 11(a) is similar to Fig. 2, which is obtained from a traveling-wave-type parametric amplifier. However, the resonant amplifier uses only four $L C$ sections and provides much higher gain due to resonance, resulting from the robustness to the pump loss effect compared to Fig. 7.

\section{Squeezing Factor}

Finally, the minimum squeezing factor can be simply calculated based on the squeezing effect, assuming that the noise of the resonator itself is negligible. This is similar to calculating the NF of a conventional LNA, where we assume that the effect of the resonator loss is negligible and that the NF is dominated by the channel noise. ${ }^{1}$ Under this assumption, the squeezing factor is

$$
S_{F}=K_{s} \cdot N F_{0}
$$

${ }^{1}$ For typical losses of on-chip passive components, this assumption is valid. Interested readers can use a similar approach shown in (39) to calculate NF when the resonator loss cannot be neglected. 


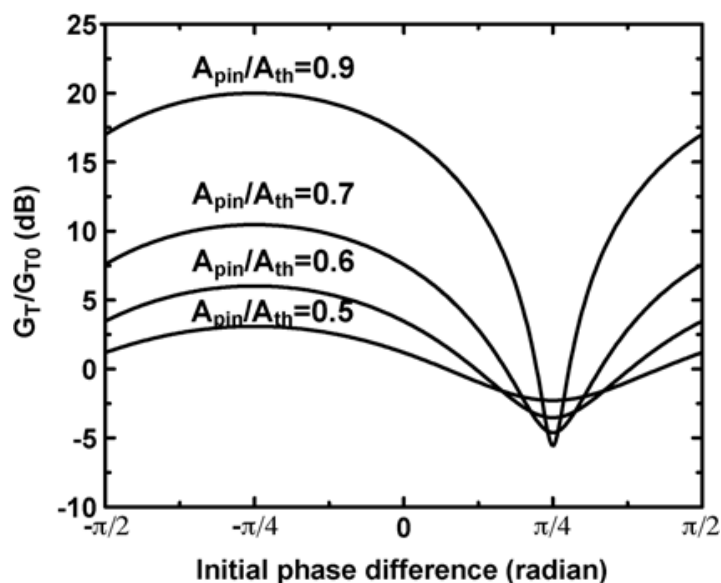

(a)

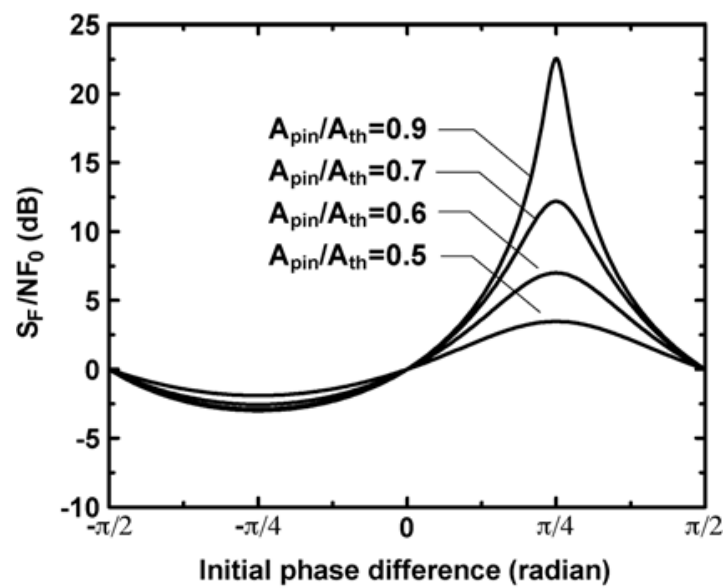

(b)

Fig. 11. Calculated (a) gain enhancement and (b) noise reduction ratios with respect to the phase difference for different pump amplitudes.

where $K_{s}$ is the noise reduction ratio and $N F_{0}$ is the NF of the source-degenerated common-source amplifier. The noise reduction ratio $K_{s}$ can be calculated using (30) and (51)

$$
K_{s}=\frac{1}{2}\left[1+\left(\frac{\left.G_{T}\right|_{\min }}{\left.G_{T}\right|_{\max }}\right)^{2}\right]=\frac{\left(\frac{A_{\mathrm{pin}}}{A_{\mathrm{th}}}\right)^{2}+1}{\left(\left(\frac{A_{\mathrm{pin}}}{A_{\mathrm{th}}}\right)+1\right)^{2}} .
$$

When $A_{\text {pin }}$ approaches $A_{\mathrm{th}}$, the reduction ratio $K_{s}$ approaches $1 / 2$.

Fig. 11(b) shows the calculated squeezing factor with respect to the phase difference between the signal and the pump for different pump amplitudes based on (55). As expected in the gain plot, a higher pump amplitude provides a higher noisesqueezing ratio for the in-phase component, whereas it degrades the noise performance of the out-of-phase component.

\section{DESIGN AND SimUlation}

We designed and simulated the proposed amplifier at around $10 \mathrm{GHz}$ in a 65-nm CMOS technology. The chip consumes 30 $\mathrm{mW}$ from a 1.2-V supply. This power includes the pump generation circuit, which consumes $14.5 \mathrm{~mW}$. The estimated area of the entire chip is $1.5 \mathrm{~mm} \times 0.9 \mathrm{~mm}$.

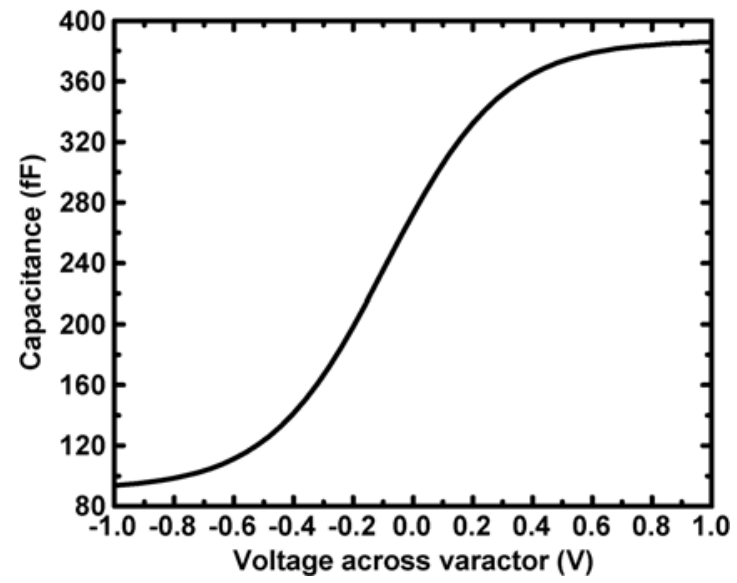

Fig. 12. Accumulation-mode MOS varactor characteristic in the $65-\mathrm{nm}$ process.

\section{A. Design}

The design of the proposed amplifier consists of three parts: a nonlinear resonator for the phase-sensitive gain, an LNA for the input stage, and a frequency doubler to generate a pump frequency that has exactly twice the input frequency. To design the resonator, we use the fact that its resonant frequency, which should be around $10 \mathrm{GHz}$, is closely related to the propagation constant $\beta$, defined by $\beta=\omega \sqrt{l c_{0}}=2\left(\omega / \omega_{c}\right)$. Also, as explained in Section II, the cutoff frequency of the $L C$ ladder sets the maximum gain of the amplifier. The optimum cutoff frequency can be obtained from the tradeoff between $\widetilde{s_{0}}$ in (20) and the pump loss due to the cutoff frequency, which is comparable to the pump frequency. Using an optimum cutoff frequency of around $25 \mathrm{GHz}$ for the maximum gain, we need a fine adjustment to satisfy the resonant frequency of $10 \mathrm{GHz}$. The resonant frequency is determined from

$$
d=n h=\frac{\lambda_{s}}{2}=\frac{\pi}{\beta}=\frac{1}{2 f_{\mathrm{osc}} \sqrt{\left(\frac{l}{h}\right)\left(\frac{c_{0}}{h}\right)}}
$$

where $d$ is the length of the resonator, $h$ is the spacing between two adjacent nodes, and $n$ is the number of resonator sections. The varactor capacitance per unit section is approximated by its average value $c_{0}$. Equation (57) can be rearranged to

$$
f_{\mathrm{osc}}=\frac{1}{2 n \sqrt{l c_{0}}}=\frac{\pi f_{c}}{2 n} .
$$

Inserting the cutoff frequency into (58), the section number is around 3.9. Because we can only have positive integers, the section number is set to four, which requires the minor adjustment of the cutoff frequency to keep the resonant frequency at $10 \mathrm{GHz}$ (for an accurate adjustment, the resonant frequency should be verified with Cadence simulation since (57) and (58) are based on the small dispersion assumption). Knowing the cutoff frequency of the line and the number of sections, we can determine the $L C$ product. To pick the values of the inductors and the varactors, we need to select the characteristic impedance of the $L C$ line. Since the resonator is driven with an LNA and since the output is taken out using a buffer, the impedance does not have to be $50 \Omega$. As a result, we select the characteristic impedance to minimize the loss of the resonator, which is around $40 \Omega$. In this 


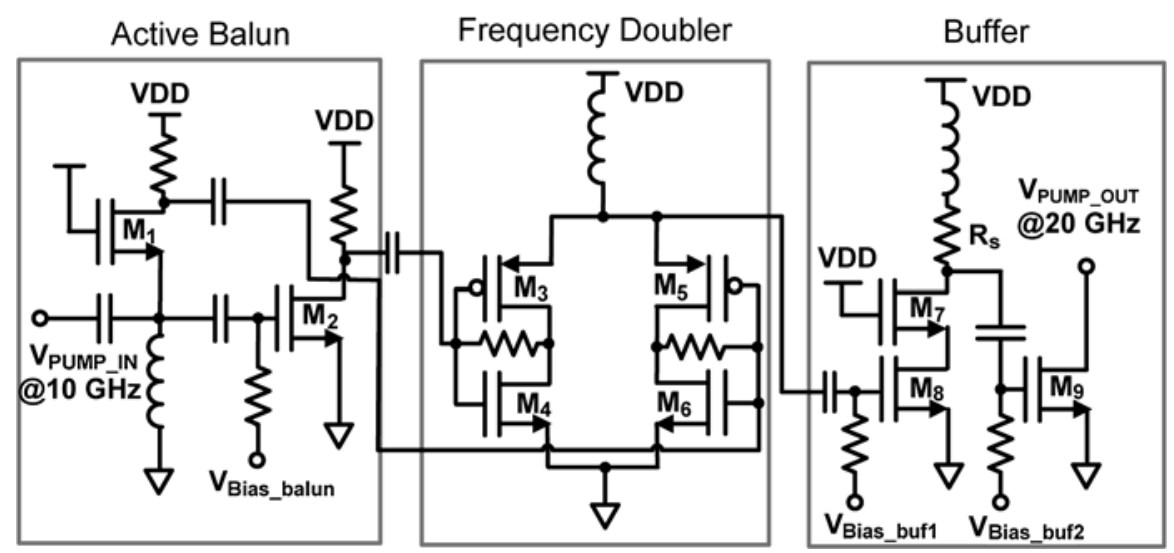

Fig. 13. Schematic of the pump generation block consisting of active balun, frequency doubler, and buffer.

process, the optimized inductor and varactor values that are used to achieve the maximum gain (i.e., optimum cutoff frequency) and minimum loss are $470 \mathrm{pH}$ and $270 \mathrm{fF}$, respectively.

The inductor is implemented as a spiral whose inductance is $380 \mathrm{pH}$ and whose dimension is $145 \mu \mathrm{m} \times 145 \mu \mathrm{m}$, including the guard ring. The quality factors of the spiral are 16 and 19 at 10 and $20 \mathrm{GHz}$, respectively. The coplanar waveguide with a ground-shielded plane is employed to connect the inductors. The inductance of these interconnects is around $90 \mathrm{pH}$. The transmission line structure is carefully simulated in an $\mathrm{E} / \mathrm{M}$ simulator (SONNET).

We use an accumulation-mode MOS varactor as a voltage-dependent capacitor. Its capacitance versus voltage characteristic is shown in Fig. 12. The average capacitance is $270 \mathrm{fF}$, and the linearized $C / V$ slope in (1) (b) is approximated to be 1.4 around a zero bias voltage. The quality factors of the varactor are 31 and 15 at 10 and $20 \mathrm{GHz}$, respectively. The varactors use the multifinger gate structure to optimize the nonlinearity and the quality factor simultaneously [29]. Metal-insulator-metal capacitors (MIMCAPs) are used for the dispersion compensation capacitors.

The design of the LNA for the input stage follows a general source-degenerated amplifier for an input frequency of $10 \mathrm{GHz}$. The input impedance is matched to $50 \Omega$. The main transistor ( $M_{1}$ in Fig. 9) has a width of $96 \mu \mathrm{m}$ for optimum NF and input matching with a bias current of $3 \mathrm{~mA}$. The width of the cascode transistor $\left(M_{2}\right)$ is selected by considering the tradeoff between the amount of parasitic capacitances and its noise contribution. In our design, the width of $M_{2}$ is $80 \mu \mathrm{m}$. Both $M_{1}$ and $M_{2}$ have a minimum channel length of $60 \mathrm{~nm}$.

To generate the pump at twice the signal frequency, the frequency doubler is implemented as shown in Fig. 13. It consists of an active balun, a frequency doubler, and a buffer. Since the frequency doubler is designed for a differential input signal, an active balun is employed. The active balun $\left(M_{1}-M_{2}\right.$ in Fig. 13) is the combination of the common-gate and common-source amplifiers to simultaneously generate the noninverting and inverting outputs [34]. In addition, the common-gate amplifier located in the input port provides a broadband input matching. The frequency doubler $\left(M_{3}-M_{6}\right)$ uses the nonlinearity of the transistor. The even-order harmonics of the differential input signal (mainly, the second-order harmonic) are picked at the common node through the resonant network. The last stage $\left(M_{7}-M_{9}\right)$ is

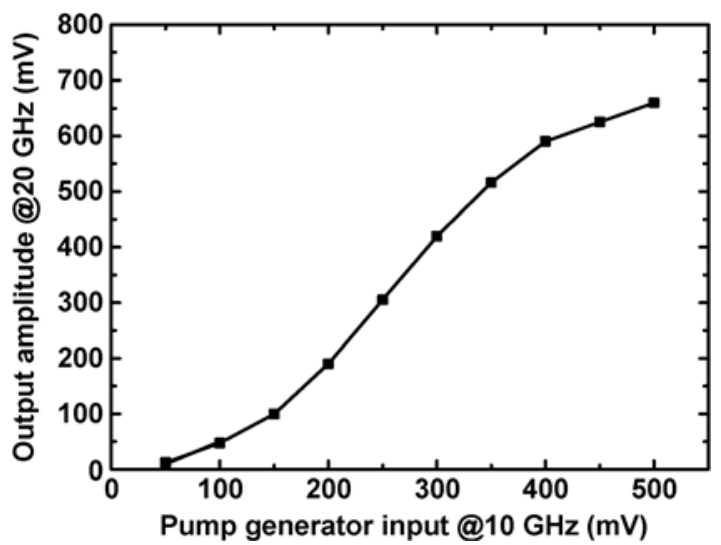

Fig. 14. Simulated frequency-doubled output of the pump generation block versus the input amplitude.

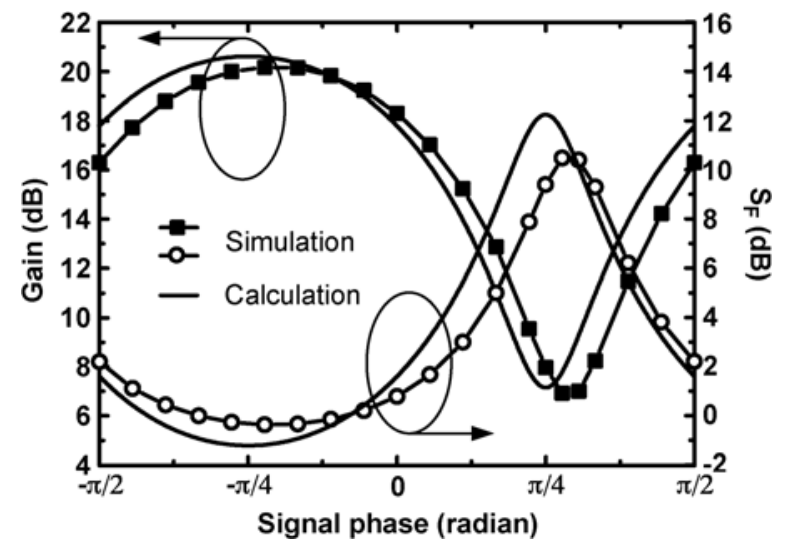

Fig. 15. Calculated and simulated gains and squeezing factors versus the signal phase in the proposed amplifier for a fixed pump amplitude $(500 \mathrm{mV})$.

a two-stage amplifier that is used as a buffer. The first stage amplifies the signal with a high gain, and the second stage drives the nonlinear resonator. Fig. 14 shows the simulated output amplitude of the pump generation block versus an input amplitude in Cadence.

\section{B. Simulation}

Using Cadence, we simulated our designed amplifier, which consists of the input stage amplifier and the nonlinear resonator. The input of the amplifier is a $10-\mathrm{GHz}$ signal (carrier) with a bandwidth of $500 \mathrm{MHz}$ (data). The pump frequency is fixed at $20 \mathrm{GHz}$, which is twice the carrier frequency. The outputs 


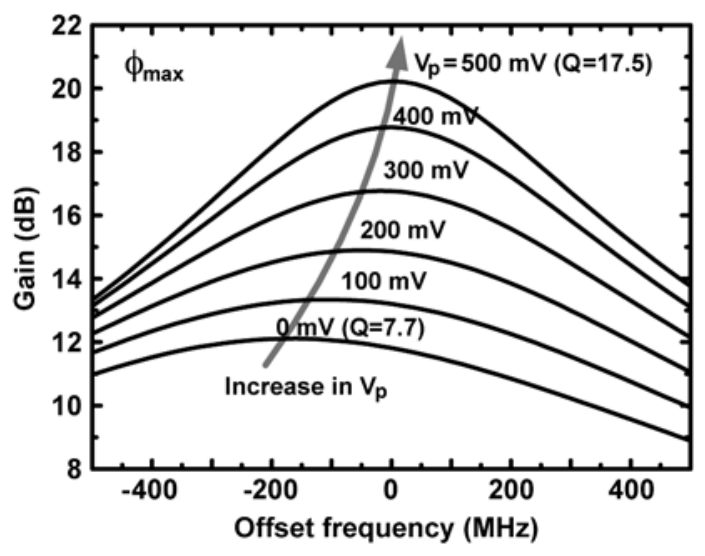

(a)

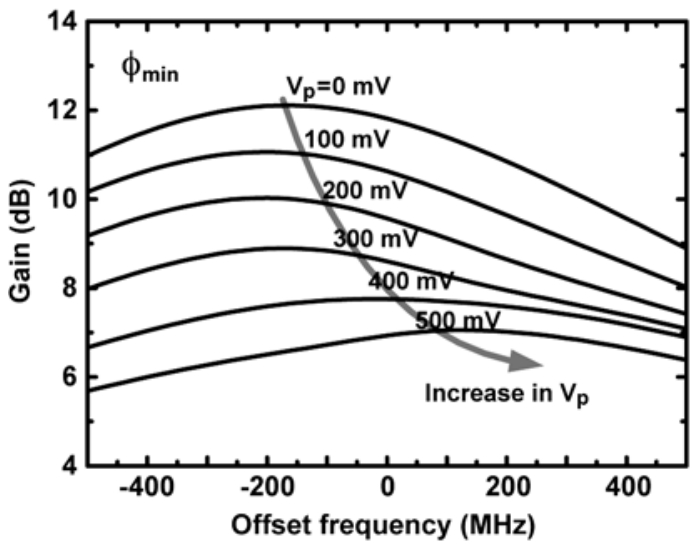

(b)

Fig. 16. Cadence simulation of the gain versus the frequency for different pump amplitudes for the (a) maximum and (b) minimum gains.

are connected to $50-\Omega$ loads using the source follower buffers. The simulation is carried out at a schematic level, including an interconnect transmission line model verified by SONNET. We use periodic noise analysis (Pnoise) and periodic S-parameter analysis (PSP) in Spectre to simulate the gain and squeezing factor of the proposed design.

Fig. 15 shows both the calculated and simulated gains and squeezing factors versus the signal phase for a fixed pump amplitude of $500 \mathrm{mV}$. For the calculation, we insert $G_{T 0}=11.5 \mathrm{~dB}$ and $N F_{0}=1.6 \mathrm{~dB}$ into (54) and (55) based on the Cadence simulation. $A_{\text {pin }} / A_{\text {th }}$ is set to 0.65 for a best fit with the simulation. Note that the accurate calculation of $A_{\mathrm{th}}$ is difficult due to the voltage-sensitive drain node impedance of the input amplifier. This is because the standing wave formation of the pump signal doubles the input pump voltage swing on the drain node, as shown in Figs. 8 and 9.

The gain ranges from 7 to $20 \mathrm{~dB}$ depending on the phase difference between the signal and the pump. The difference between the maximum and minimum gains is around $13 \mathrm{~dB}$, which determines the magnitude of the noise-squeezing ratio in (55). As expected in (54), the plots are periodic with a period of $\pi$, and the signal phase difference between the maximum and minimum gains is $\pi / 2$, implying a quadrature squeezing. The simulated gain includes a 6-dB loss due to the output buffer, which is not calibrated (the maximum gain is $26 \mathrm{~dB}$ before the buffer, whereas the graph in Fig. 15 shows $20 \mathrm{~dB}$ at its maximum). The squeezing factor plot is the flip-down image of the gain plot,

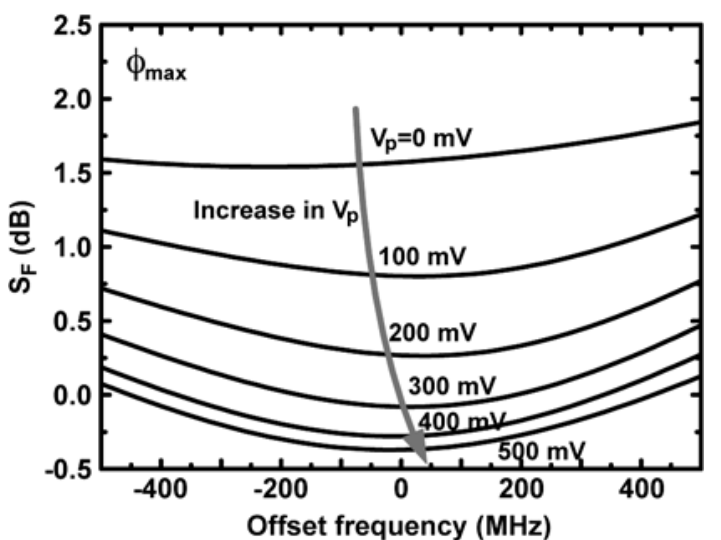

(a)

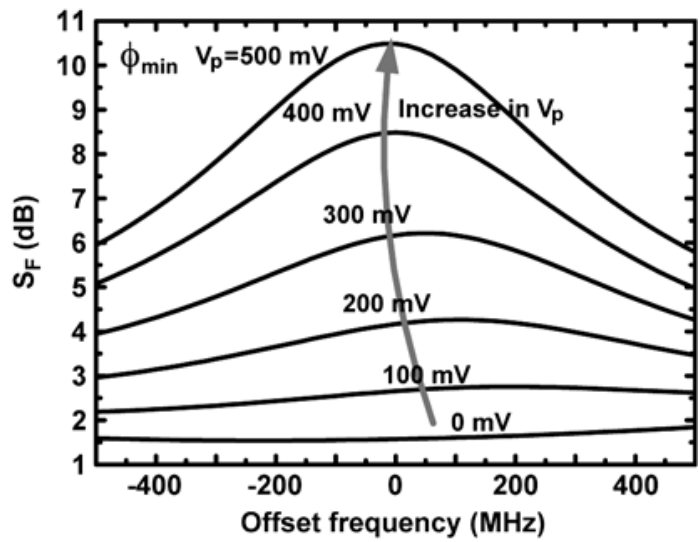

(b)

Fig. 17. Cadence simulation of the squeezing factor versus the frequency for different pump amplitudes for the (a) maximum and (b) minimum gains.

as we observed in Section III. Assuming that all of the signals are in-phase components, the noise-squeezing effect suppresses the squeezing factor even below $0 \mathrm{~dB}$, resulting in a minimum squeezing factor of $-0.37 \mathrm{~dB}$ when the gain is maximum. From (55) and (56), the theoretical squeezing factor is suppressed up to $3 \mathrm{~dB}$ below the NF of the input LNA as the pump input gets close to the oscillation threshold. However, the simulation result shows a 2-dB suppression of the squeezing factor from 1.6 to $-0.37 \mathrm{~dB}$. This is because the additional loss from the drain node of the input amplifier increases the threshold voltage beyond the varactor saturation region, where the capacitance does not change with the voltage, as shown in Fig. 12.

We also simulated the gain and squeezing factor for different pump amplitudes with a fixed signal phase, particularly for the maximum and minimum gain cases, as shown in Figs. 16 and 17. In the maximum gain case, the increase in pump amplitude boosts the gain by increasing the quality factor of the resonator. The increase in the quality factor can also be verified in Fig. 16(a), as well as by observing that the bandwidth becomes narrower with the pump amplitude. Fig. 17(a) shows that the squeezing factor is more suppressed as the difference between the maximum and minimum gains increases with the pump amplitude due to the squeezing effect. On the other hand, Fig. 16(b) shows that the increase in the pump amplitude decreases the signal gain for a phase at minimum gain. The destructive adding process causes more signal attenuation inside the resonator with the pump amplitude, degrading the quality 


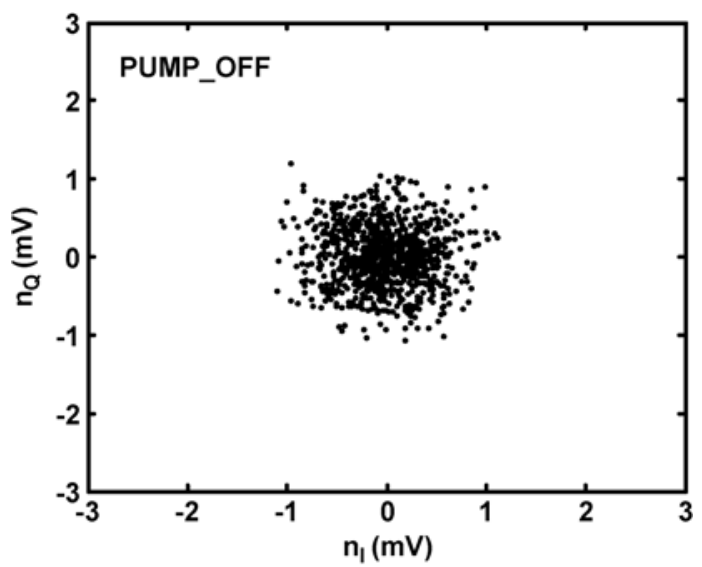

(a)

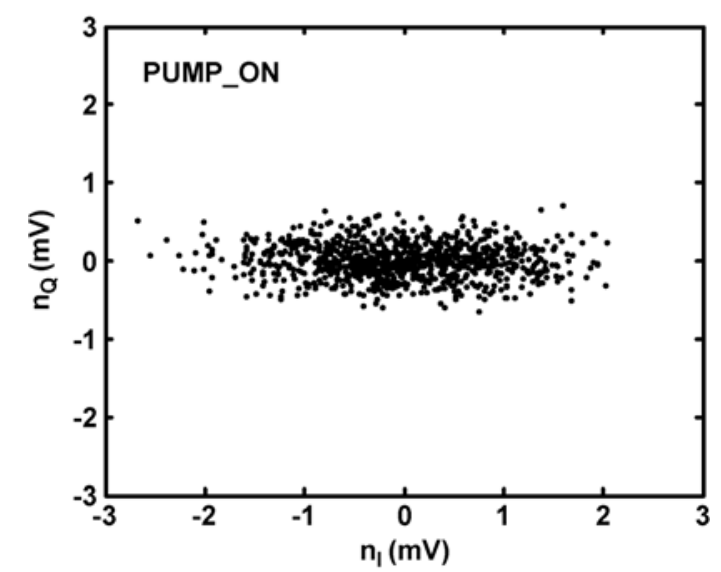

(b)

Fig. 18. Cadence simulation of the noise-squeezing effect (a) for a zero pump amplitude and (b) for a pump amplitude of $500 \mathrm{mV}$.

factor. The squeezing factor also becomes worse due to signal loss, as shown in Fig. 17(b).

The noise-squeezing effect is also clearly observed using the transient noise option in the transient analysis in Cadence, as shown in Fig. 18. In the simulation setup, two independent noise voltages, whose bandwidth is $500 \mathrm{MHz}$, are generated and combined though $I / Q$ modulation at a $10-\mathrm{GHz}$ LO frequency. The combined noise signal is injected to the resonant parametric amplifier. After passing through the proposed amplifier, the output is demodulated to extract the $I / Q$ components and is sampled for statistical analysis. In the transient simulation, it is noted that the intrinsic device noise is neglected due to a large-signal input noise source. Fig. 18 shows the simulated noise squeezing, depending on the presence of the pump. Before the pump injection, the output noise distribution is still circularly symmetric for the in-phase and out-of-phase components (hence, it is phase insensitive). However, the out-of-phase noise component is suppressed in the presence of the pump signal, whereas the in-phase noise component is amplified. Note that the input signal is also equally amplified with the in-phase noise component. To quantitatively analyze the suppressed out-of-phase noise component, the histogram is plotted for 960 samples, as shown in Fig. 19. We clearly observe that the standard deviation of the out-of-phase noise component is significantly suppressed with pump injection.

Linearity is simulated in terms of the compression characteristics and the input-referred IP3. Fig. 20 shows that the 1-dB

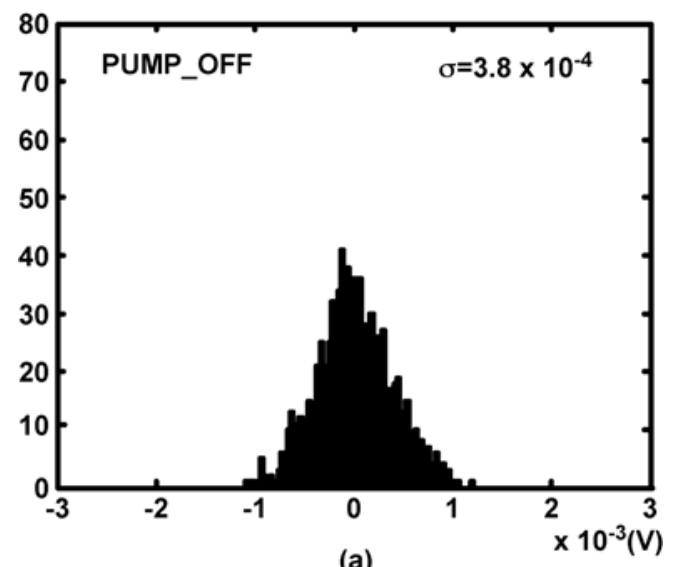

(a)

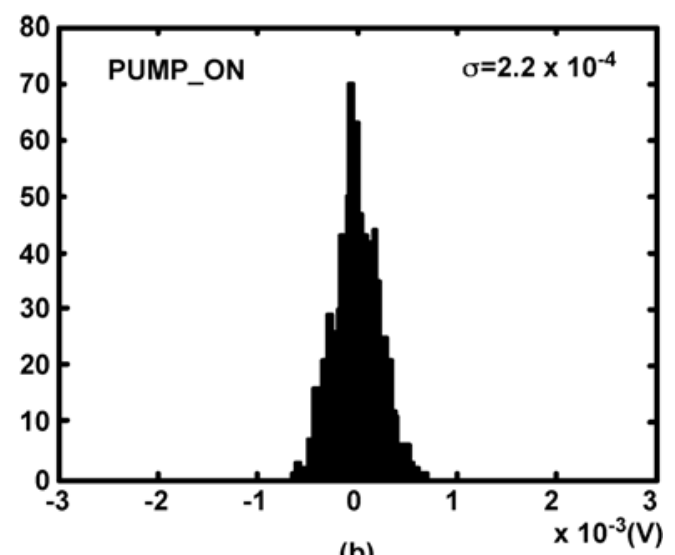

(b)

Fig. 19. Simulated noise histogram (a) for a zero pump amplitude and (b) for a pump amplitude of $500 \mathrm{mV}$ using Cadence.

output compression point is $-2.9 \mathrm{dBm}$ for the proposed amplifier when the pump is not injected. However, when the pump amplitude is $500 \mathrm{mV}$, the $1-\mathrm{dB}$ output compression point decreases by around $3 \mathrm{~dB}$ since the gain partially comes from the limited power of the pump. The proposed amplifier provides an IIP3 ranging from -7.2 to $-8 \mathrm{dBm}$ depending on the pump amplitude, as shown in Fig. 21. For comparison, the linear modification of the proposed amplifier is also simulated after replacing all varactors with MIMCAPs in the resonator. The $1-\mathrm{dB}$ output compression point and IIP3 are simulated to be -2.2 and $-6.3 \mathrm{dBm}$ for the linear modification, respectively. Even though the proposed amplifier employs the nonlinear resonator, the degradation in linearity performance is not large compared to its linear version. This is because the parametric resonator mainly generates the second-order nonlinearity due to the first-order $C / V$ characteristic of the varactor, while both IIP3 and gain compression are caused by the third-order nonlinearity. IIP2, caused by the second-order nonlinearity, is not considered here since it is not problematic for a narrow-band amplifier like the proposed one.

\section{CONCLUSION}

We have proposed a resonant parametric amplifier with a low-noise performance using the noise-squeezing effect. The phase-sensitive amplification process suppresses one of the quadrature noise components and achieves around 3-dB noise reduction for a single-phase information compared to the 


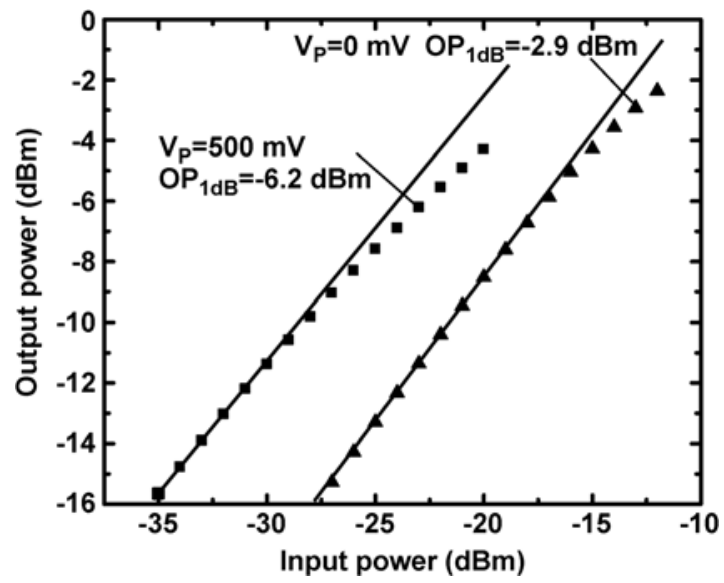

Fig. 20. Cadence simulation of the gain compression for a zero pump amplitude and for a pump amplitude of $500 \mathrm{mV}$.

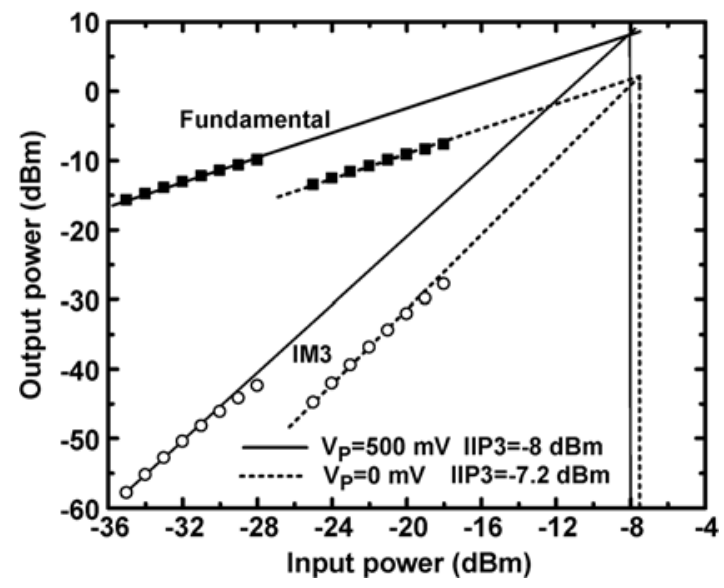

Fig. 21. Cadence simulation of the input-referred IP3 for a zero pump amplitude and for a pump amplitude of $500 \mathrm{mV}$.

phase-insensitive amplification. The resonant structure of the amplifier, which resembles the Fabry-Perot laser amplifier, enables parametric amplification for narrow-band signals with a small number of lumped $L C$ elements. The signal gain and squeezing factor are theoretically analyzed based on the continuous transmission line approximation and on the steady-state assumption. We have achieved a minimum squeezing factor of $-0.35 \mathrm{~dB}$ for a $10-\mathrm{GHz}$ narrow-band signal in Cadence simulation.

\section{ACKNOWLEDGMENT}

The authors would like to thank G. Li, O. Momeni, Y. Tousi, M. Adnan, E. Hwang, Prof. Apsel, Prof. Molnar, and Prof. Pollock of Cornell University for the helpful discussions regarding various aspects of this paper and M. Azarmnia and $\mathrm{H}$. Yu for their support.

\section{REFERENCES}

[1] D. K. Shaeffer and T. H. Lee, "A 1.5-V, 1.5-GHz CMOS low noise amplifier," IEEE J. Solid-State Circuits, vol. 32, no. 5, pp. 745-759, May 1997.

[2] A. Nieuwoudt, T. Ragheb, H. Nejati, and Y. Massoud, "Numerical design optimization methodology for wideband and multi-band inductively degenerated cascode CMOS low noise amplifiers," IEEE Trans. Circuits Syst. I, Reg. Papers, vol. 56, no. 6, pp. 1088-1101, Jun. 2009.
[3] X. Li, S. Shekhar, and D. J. Allstot, " $G_{m}$-boosted common-gate LNA and differential colpitts $\mathrm{VCO} / \mathrm{QVCO}$ in 0.18- $\mu \mathrm{m} \mathrm{CMOS,"} \mathrm{IEEE} J$. Solid-State Circuits, vol. 40, no. 12, pp. 2609-2619, Dec. 2005.

[4] A. Liscidini, M. Brandolini, D. Sanzogni, and R. Castello, "A 0.13 $\mu \mathrm{m}$ CMOS front-end for DCS1800/UMTS/802.11b-g with multi-band positive feedback low noise amplifier," IEEE J. Solid-State Circuits, vol. 41, no. 4, pp. 981-989, Apr. 2006.

[5] L. Belostotski and J. W. Haslett, "Sub-0.2 dB noise figure wideband room-temperature CMOS LNA with non-50 $\Omega$ signal-source impedance," IEEE J. Solid-State Circuits, vol. 42, no. 11, pp. 2492-2502, Nov. 2007.

[6] J. M. Manley and H. E. Rowe, "Some general properties of nonlinear elements-Part I. General energy relations," Proc. IRE, vol. 44, no. 7, pp. 904-913, Jul. 1956.

[7] S. Ranganathan and Y. Tsividis, "A MOS capacitor-based discretetime parametric amplifier with $1.2 \mathrm{~V}$ output swing and $3 \mu \mathrm{W}$ power dissipation," in Proc. IEEE ISSCC Dig. Tech. Papers, Feb. 2003, pp. 406-407.

[8] S. Ranganathan and Y. Tsividis, "Discrete-time parametric amplification based on a three-terminal MOS varactor: Analysis and experimental results," IEEE J. Solid-State Circuits, vol. 38, no. 12, pp. 2087-2093, Dec. 2003.

[9] P. M. Figueiredo and J. C. Vital, "The MOS capacitor amplifier," IEEE Trans. Circuits Syst. II, Exp. Briefs, vol. 51, no. 3, pp. 111-115, Mar. 2004.

[10] S. Magierowski, H. Chan, and T. Zourntos, "Subharmonically pumped RF CMOS paramps," IEEE Trans. Electron Devices, vol. 55, no. 2, pp. 601-608, Feb. 2008

[11] S. Magierowski, T. Zourntos, J. Bousquet, and Z. Zhao, "Compact parametric downconversion using MOS varactors," in Proc. IEEE Int. Microw. Symp., Jun. 2009, pp. 1377-1380.

[12] W. Lee and E. Afshari, "Parametric distributed resonator: A passive frequency divider," IEEE J. Solid-State Circuits, vol. 45, no. 9, pp. 1834-1844, Sep. 2010.

[13] Y. Yamamoto, "Characteristics of AlGaAs Fabry-Perot cavity type laser amplifiers," IEEE J. Quantum Electron., vol. QE-16, no. 10, pp. $1047-1052$, Oct. 1980.

[14] K. G. Köprülü and O. Aytür, "Analysis of the generation of amplitudesqueezed light with Gaussian-beam degenerate optical parametric amplifiers," J. Opt. Soc. Amer. B, Opt. Phys., vol. 18, no. 6, pp. 846-854, Jun. 2001.

[15] T. Hirano, K. Kotani, T. Ishibashi, S. Okude, and T. Kuwamoto, "3 $\mathrm{dB}$ squeezing by single-pass parametric amplification in a periodically poled $\mathrm{KTiOPO}_{4}$ crystal," Opt. Lett., vol. 30, no. 13, pp. 1722-1724, Jul. 2005.

[16] D. Rugar, "Mechanical parametric amplification and thermomechanical noise squeezing," Phys. Rev. Lett., vol. 67, no. 6, pp. 699-702, Aug. 1991.

[17] R. Almog, "Noise squeezing in a nanomechanical duffing resonator," Phys. Rev. Lett., vol. 98, no. 7, p. 078 103, Feb. 2007, 10.1103/PhysRevLett.98.078103.

[18] B. Yurke, "Squeezed-state generation using a Josephson parametric amplifier," J. Opt. Soc. Amer. B, Opt. Phys., vol. 4, no. 10, pp. 1551-1557, Oct. 1987

[19] B. Yurke, "Observation of 4.2-K equilibrium-noise squeezing via a Josephson-parametric amplifier," Phys. Rev. Lett., vol. 60, no. 9, pp. 764-767, Feb. 1988.

[20] M. A. Castellanos-Beltran, K. D. Irwin, G. C. Hilton, L. R. Vale, and $\mathrm{K}$. W. Lehnert, "Amplification and squeezing of quantum noise with a tunable Josephson metamaterial," Nature, vol. 4, no. 12, pp. 929-931, Oct. 2008, 10.1038/nphys 1090 .

[21] E. Afshari and A. Hajimiri, "Nonlinear transmission lines for pulse shaping in silicon," IEEE J. Solid-State Circuits, vol. 40, no. 3, pp. 744-752, Mar. 2005.

[22] W. H. Louisell, Coupled Mode and Parametric Electronics. New York: Wiley, 1960.

[23] P. K. Tien and H. Suhl, "A traveling-wave ferromagnetic amplifier," Proc. IRE, vol. 46, no. 4, pp. 700-706, Apr. 1958.

[24] P. K. Tien, "Parametric amplification and frequency mixing in propagating circuits," J. Appl. Phys., vol. 29, no. 9, pp. 1347-1357, Sep. 1958.

[25] D. M. Pozar, Microwave Engineering. New York: Wiley, 2005.

[26] J. J. Waterston, "On the physics of media that are composed of free and elastic molecules in a state of motion," Roy. Soc. Proc., vol. 5, p. 604, 1846.

[27] W. Greiner, L. Neise, and H. Stocker, Thermodynamics and Statistical Mechanics. New York: Springer-Verlag, 1995. 
[28] R. F. Wiser, M. Zargari, D. K. Su, and B. A. Wooley, "A 5-GHz wireless LAN transmitter with integrated tunable high- $Q$ RF filter," IEEE J. Solid-State Circuits, vol. 44, no. 8, pp. 2114-2125, Aug. 2009.

[29] C.-C. Ho, C.-W. Kuo, Y.-J. Chan, W.-Y. Lien, and J.-C. Guo, "0.13$\mu \mathrm{m}$ RF CMOS and varactors performance optimization by multiple gate layouts," IEEE Trans. Electron Devices, vol. 51, no. 12, pp. 2181-2185, Dec. 2004.

[30] "CMRF8SF Model Reference Guide," IBM Microelectronics Division, New York, Apr. 2007.

[31] L. E. Myers, R. C. Eckardt, M. M. Fejer, and R. L. Byer, "Quasi-phase-matched optical parametric oscillators in bulk periodically poled $\mathrm{LiNbO}_{3}$, , J. Opt. Soc. Amer. B, Opt. Phys., vol. 12, no. 11, pp. 2102-2116, Nov. 1995.

[32] G. M. Roe and M. R. Boyd, "Parametric energy conversion in distributed systems," Proc. IRE, vol. 47, no. 7, pp. 1213-1218, Jul. 1959.

[33] R. Landauer, "Parametric amplification along nonlinear transmission lines," J. Appl. Phys., vol. 31, no. 3, pp. 479-484, Mar. 1960.

[34] R. Bagheri, A. Mirzaei, S. Chehrazi, M. E. Heidari, M. Lee, M. Mikhemar, W. Tang, and A. A. Abidi, "An $800-\mathrm{MHz}-6-\mathrm{GHz}$ software-defined wireless receiver in 90-nm CMOS," IEEE J. Solid-State Circuits, vol. 41, no. 12, pp. 2860-2876, Dec. 2006.

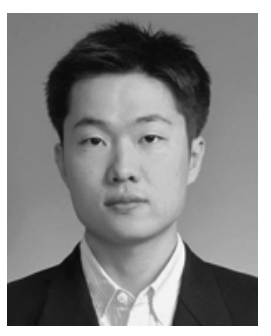

Wooram Lee (S'07) received the B.Sc. and M.S. degrees in electrical engineering from the Korea Advanced Institute of Science and Technology, Daejeon, Korea, in 2001 and 2003, respectively. $\mathrm{He}$ is currently working toward the Ph.D. degree at Cornell University, Ithaca, NY.

He was a Research Engineer with the Electronics and Telecommunications Research Institute, Daejeon, where he worked on optical transceivers and links for wavelength-division-multiplexed passive optical network (WDM-PON) from 2003 to 2007. His research interests include high-performance RFIC design based on nonlinear electronics for low-noise parametric amplification, oscillation, and terahertz pulse generation.

Mr. Lee received the IEEE Solid-State Circuits Predoctoral Fellowship for 2010-2011 and the Samsung Graduate Fellowship for 2007-2011. He was also a recipient of the Best Paper Award of the IEEE Radar Conference in 2009 and the Silver Medal at the National Physics Competition in 1996.

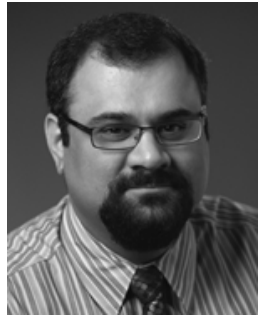

Ehsan Afshari (S'98-M'07) was born in 1979. He received the B.Sc. degree in electronics engineering from the Sharif University of Technology, Tehran, Iran, and the M.S. and Ph.D. degrees in electrical engineering from the California Institute of Technology, Pasadena, in 2003 and 2006, respectively.

In August 2006, he joined the Faculty in Electrical and Computer Engineering, Cornell University, Ithaca, NY. His research interests include high-speed and low-noise integrated circuits for applications in devices communication systems, sensing, and biomedical

Prof. Afshari serves as the Chair of the IEEE Ithaca section, the Chair of Cornell Highly Integrated Physical Systems, and a member of the Analog Signal Processing Technical Committee of the IEEE Circuits and Systems Society. He was the recipient of Iran's Best Engineering Student award by the President of Iran in 2001, the Defense Advanced Research Projects Agency Young Faculty Award in 2008, and the National Science Foundation CAREER award in 2010. He was also the recipient of the Silver Medal in the Physics Olympiad in 1997, the Best Undergraduate Paper Award in the Iranian Conference on Electrical Engineering in 1999, the Best Paper Award in the Custom Integrated Circuits Conference in September 2003, the Award of Excellence in Engineering Education from the Association of Professors and Scholars of Iranian Heritage in May 2004, and the First Place at the Stanford-Berkeley-Caltech Inventors Challenge in March 2005. 